\title{
Prismatic large $N$ models for bosonic tensors
}

\author{
Simone Giombi, ${ }^{1}$ Igor R. Klebanov, ${ }^{1,2}$ Fedor Popov, ${ }^{1}$ Shiroman Prakash, ${ }^{3}$ and Grigory Tarnopolsky ${ }^{4}$ \\ ${ }^{1}$ Department of Physics, Princeton University, Princeton, New Jersey 08544, USA \\ ${ }^{2}$ Princeton Center for Theoretical Science, Princeton University, Princeton, New Jersey 08544, USA \\ ${ }^{3}$ Department of Physics and Computer Science, Dayalbagh Educational Institute, Agra 282005, India \\ ${ }^{4}$ Department of Physics, Harvard University, Cambridge, Massachusetts 02138, USA
}

(Received 5 September 2018; published 14 November 2018)

\begin{abstract}
We study the $O(N)^{3}$ symmetric quantum field theory of a bosonic tensor $\phi^{a b c}$ with sextic interactions. Its large $N$ limit is dominated by a positive-definite operator, whose index structure has the topology of a prism. We present a large $N$ solution of the model using Schwinger-Dyson equations to sum the leading diagrams, finding that for $2.81<d<3$ and for $d<1.68$ the spectrum of bilinear operators has no complex scaling dimensions. We also develop perturbation theory in $3-\epsilon$ dimensions including eight $O(N)^{3}$ invariant operators necessary for the renormalizability. For sufficiently large $N$, we find a "prismatic" fixed point of the renormalization group, where all eight coupling constants are real. The large $N$ limit of the resulting $\epsilon$ expansions of various operator dimensions agrees with the SchwingerDyson equations. Furthermore, the $\epsilon$ expansion allows us to calculate the $1 / N$ corrections to operator dimensions. The prismatic fixed point in $3-\epsilon$ dimensions survives down to $N \approx 53.65$, where it merges with another fixed point and becomes complex. We also discuss the $d=1$ model where our approach gives a slightly negative scaling dimension for $\phi$, while the spectrum of bilinear operators is free of complex dimensions.
\end{abstract}

DOI: 10.1103/PhysRevD.98.105005

\section{INTRODUCTION}

In recent literature, there has been considerable interest in models where the degrees of freedom transform as tensors of rank 3 or higher. Such models with appropriately chosen interactions admit new kinds of large $N$ limits, which are not of 't Hooft type and are dominated by the socalled melonic Feynman diagrams [1-5]. Much of the recent activity (for a review see [6]) has been on the quantum mechanical models of fermionic tensors $[4,5]$, which have large $N$ limits similar to that in the Sachdev-YeKitaev (SYK) model [7-14].

It is also of interest to explore similar quantum theories of bosonic tensors $[5,15,16]$. In $[5,15]$ an $O(N)^{3}$ invariant theory of the scalar fields $\phi^{a b c}$ was studied:

$$
\begin{aligned}
S_{4} & =\int d^{d} x\left(\frac{1}{2}\left(\partial_{\mu} \phi^{a b c}\right)^{2}+\frac{g}{4 !} O_{\text {tetra }}\right), \\
O_{\text {tetra }} & =\phi^{a_{1} b_{1} c_{1}} \phi^{a_{1} b_{2} c_{2}} \phi^{a_{2} b_{1} c_{2}} \phi^{a_{2} b_{2} c_{1}} .
\end{aligned}
$$

This quantum field theory (QFT) is super-renormalizable in $d<4$ and is formally solvable using the Schwinger-Dyson

Published by the American Physical Society under the terms of the Creative Commons Attribution 4.0 International license. Further distribution of this work must maintain attribution to the author(s) and the published article's title, journal citation, and DOI. Funded by SCOAP ${ }^{3}$. equations in the large $N$ limit where $g N^{3 / 2}$ is held fixed. However, this model has some instabilities. One problem is that the "tetrahedral" operator $O_{\text {tetra }}$ is not positive definite. Even if we ignore this and consider the large $N$ limit formally, we find that in $d<4$ the $O(N)^{3}$ invariant operator $\phi^{a b c} \phi^{a b c}$ has a complex dimension of the form $\frac{d}{2}+i \alpha(d)$ [15]. ${ }^{1}$ From the dual anti-de Sitter (AdS) point of view, such a complex dimension corresponds to a scalar field whose $m^{2}$ is below the Breitenlohner-Freedman stability bound $[21,22]$. The origin of the complex dimensions was elucidated using perturbation theory in $4-\epsilon$ dimensions: the fixed point was found to be at complex values of the couplings for the additional $O(N)^{3}$ invariant operators required by the renormalizability [15]. In [15] an $O(N)^{5}$ symmetric theory for tensor $\phi^{a b c d e}$ and sextic interactions was also considered. It was found that the dimension of operator $\phi^{\text {abcde }} \phi^{\text {abcde }}$ is real in the narrow range $d_{\text {crit }}<d<3$, where $d_{\text {crit }} \approx 2.97$. However, the scalar potential of this theory is again unstable, so the theory may be defined only formally. In spite of these problems, some interesting formal results on melonic scalar theories of this type were found recently [23].

\footnotetext{
${ }^{1}$ Such complex dimensions appear in various other large $N$ theories; see, e.g., [17-20].
} 


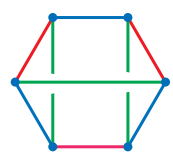

$g_{1}$

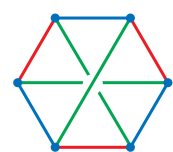

$g_{2}$

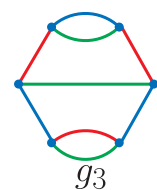

$g_{3}$

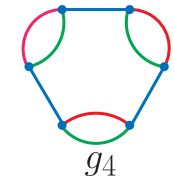

$g_{4}$

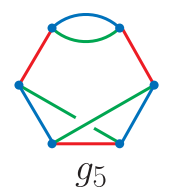

95

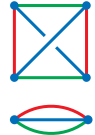

$g_{6}$

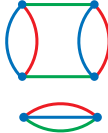

$g_{7}$

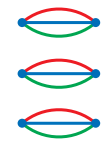

$g_{8}$

FIG. 1. Diagrammatic representation of the eight possible $O(N)^{3}$ invariant sextic interaction terms.

In this paper, we continue the search for stable bosonic large $N$ tensor models with multiple $O(N)$ symmetry groups. Specifically, we study the $O(N)^{3}$ symmetric theory of scalar fields $\phi^{a b c}$ with a sixth-order interaction, whose Euclidean action is

$$
\begin{aligned}
S_{6}= & \int d^{d} x\left(\frac{1}{2}\left(\partial_{\mu} \phi^{a b c}\right)^{2}\right. \\
& \left.+\frac{g_{1}}{6 !} \phi^{a_{1} b_{1} c_{1}} \phi^{a_{1} b_{2} c_{2}} \phi^{a_{2} b_{1} c_{2}} \phi^{a_{3} b_{3} c_{1}} \phi^{a_{3} b_{2} c_{3}} \phi^{a_{2} b_{3} c_{3}}\right) .
\end{aligned}
$$

This QFT is super-renormalizable in $d<3$. When the fields $\phi^{a b c}$ are represented by vertices and index contractions by edges, this interaction term looks like a prism (see Fig. 11 in [5]); it is the leftmost diagram in Fig. 1. Unlike with the tetrahedral quartic interaction (1.1), the action (1.2) is positive for $g_{1}>0$. In Secs. II and III, we will show that there is a smooth large $N$ limit where $g_{1} N^{3}$ is held fixed and derive formulas for various operator dimensions in continuous $d$. We will call this large $N$ limit the "prismatic" limit: the leading Feynman diagrams are not the same as the melonic diagrams, which appear in the $O(N)^{5}$ symmetric $\phi^{6}$ QFT for a tensor $\phi^{a b c d e}$ [15]. However, as we discuss in Sec. II, the prismatic interaction may be reduced to a tetrahedral one, (2.1), by introducing an auxiliary tensor field $\chi^{a b c}$.

The theory (1.2) may be viewed as a tensor counterpart of the bosonic theory with random couplings, which was introduced in Sec. VI. B of [16]. Since both theories are dominated by the same class of diagrams in the large $N$ limit, they have the same Schwinger-Dyson equations for the 2-point and 4-point functions. We will confirm the conclusion of [16] that the $d=2$ theory does not have a stable IR limit; this is due to the appearance of a complex scaling dimension. However, we find that in the ranges $2.81<d<3$ and $d<1.68$, the large $N$ prismatic theory does not have any complex dimensions for the bilinear operators. In Sec. V we use renormalized perturbation theory to develop the $3-\epsilon$ expansion of the prismatic QFT. We have to include all eight operators invariant under the $O(N)^{3}$ symmetry and the $S_{3}$ symmetry permuting the $O(N)$ groups; they are shown in Fig. 1 and written down in (A1). For $N>N_{\text {crit, }}$, where $N_{\text {crit }} \approx 53.65$, we find a prismatic RG fixed point where all eight coupling constants are real. At this fixed point, $\epsilon$ expansions of various operator dimensions agree in the large $N$ limit with those obtained using the Schwinger-Dyson equations. Furthermore, the $3-\epsilon$ expansion provides us with a method to calculate the $1 / N$ corrections to operator dimensions, as shown in (5.8), (5.9). At $N=N_{\text {crit }}$ the prismatic fixed point merges with another fixed point, and for $N<N_{\text {crit }}$ both become complex.

In Sec. VI we discuss the $d=1$ version of the model (1.2). Our large $N$ solution gives a slightly negative scaling dimension, $\Delta_{\phi} \approx-0.09$, while the spectrum of bilinear operators is free of complex scaling dimensions.

\section{LARGE $N$ LIMIT}

To study the large $N$ limit of this theory, we will find it helpful to introduce an auxiliary field $\chi^{a b c}$ so that ${ }^{2}$

$$
\begin{aligned}
S= & \int d^{d} x\left(\frac{1}{2}\left(\partial_{\mu} \phi^{a b c}\right)^{2}\right. \\
& \left.+\frac{g}{3 !} \phi^{a_{1} b_{1} c_{1}} \phi^{a_{1} b_{2} c_{2}} \phi^{a_{2} b_{1} c_{2}} \chi^{a_{2} b_{2} c_{1}}-\frac{1}{2} \chi^{a b c} \chi^{a b c}\right),
\end{aligned}
$$

where $g \sim \sqrt{g}_{1}$. Integrating out $\chi^{a b c}$ gives rise to the action (1.2). The advantage of keeping $\chi^{a b c}$ explicitly is that the theory is then a theory with $O(N)^{3}$ symmetry dominated by the tetrahedral interactions, except it now involves two rank-3 fields; this shows that it has a smooth large $N$ limit. Thus, a prismatic large $N$ limit for the theory with one 3-tensor $\phi^{a b c}$ may be viewed as a tetrahedral limit for two 3-tensors.

Let us define the following propagators:

$$
\begin{aligned}
\langle\phi(p) \phi(q)\rangle & =(2 \pi)^{d} \delta^{d}(p+q) G(p), \\
\langle\chi(p) \chi(q)\rangle & =(2 \pi)^{d} \delta^{d}(p+q) F(p) .
\end{aligned}
$$

In the free theory $G(p)=G_{0}(p)=\frac{1}{p^{2}}$, and $F(p)=F_{0}=1$. In the strong coupling limit the self-energies of the fields are given by the inverse propagators: $G(p)^{-1}=\Sigma_{\phi}$ and $F(p)^{-1}=\Sigma_{\chi}$. The Schwinger-Dyson equations for the exact two-point functions can be written as:

\footnotetext{
${ }^{2}$ If we added fermions to make the tensor model supersymmetric $[5,16,24,25]$ then $\chi^{a b c}$ would be interpreted as the highest component of the superfield $\Phi^{a b c}$.
} 


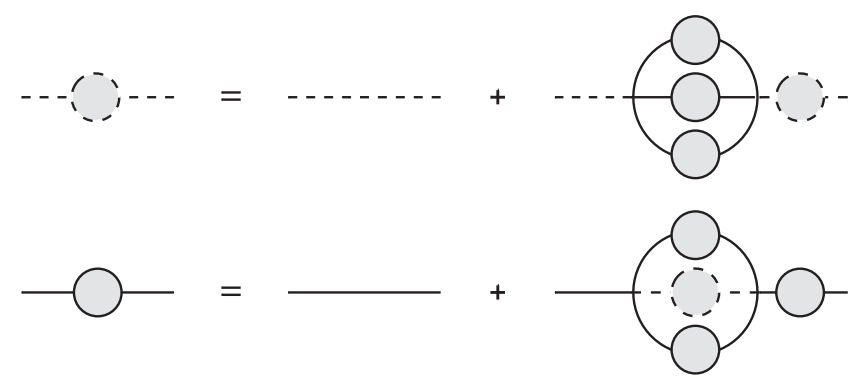

FIG. 2. Diagrammatic representation of the Schwinger-Dyson equations. Solid lines denote $\phi$ propagators, and dashed lines denote $\chi$ propagators.

$$
\begin{aligned}
F(p)= & F_{0}+g^{2} N^{3} F_{0} \int \frac{d^{d} q d^{d} k}{(2 \pi)^{2 d}} G(p-q-k) \\
& \times G(q) G(k) F(p), \\
G(p)= & G_{0}(p)+3 g^{2} N^{3} G_{0}(p) \int \frac{d^{d} q d^{d} k}{(2 \pi)^{2 d}} \\
& \times G(p-q-k) F(q) G(k) G(p),
\end{aligned}
$$

and represented in Fig. 2.

Multiplying the first equation by $F_{0}^{-1}$ on the left and $F(p)^{-1}$ on the right, and likewise for the second equation, we obtain:

$$
\begin{aligned}
& F(p)^{-1}=F_{0}^{-1}-\lambda^{2} \int \frac{d^{d} q d^{d} k}{(2 \pi)^{2 d}} G(p-q-k) G(q) G(k), \\
& G(p)^{-1}=G_{0}(p)^{-1}-3 \lambda^{2} \int \frac{d^{d} q d^{d} k}{(2 \pi)^{2 d}} G(p-q-k) F(q) G(k),
\end{aligned}
$$

where $\lambda^{2}=N^{3} g^{2} \sim N^{3} g_{1}$. We have to take the large $N$ limit keeping $\lambda^{2}$ fixed. In the IR limit, let us assume

$$
G(p)=\frac{A}{p^{2 a}}, \quad F(p)=\frac{B}{p^{2 b}}
$$

$a$ is related to the scaling dimension of $\phi, \Delta_{\phi}$ via $a=d / 2-\Delta_{\phi}$.

For what range of $a$ and $b$ can we drop the free terms in the gap equations above? In the strong coupling limit we require $b<0$ and $a<1$. Since $b=-3 a+d$, we have $d / 3<a<1$. In terms of $\Delta_{\phi}$, we then find

$$
3 \Delta_{\phi}+\Delta_{\chi}=d, \quad d / 2-1<\Delta_{\phi}<d / 6 .
$$

Notice that if we had the usual kinetic term for the $\chi$ field, the allowed range for $\Delta_{\phi}$ would be larger. Therefore, our solution may also apply to a model with two dynamical scalar fields interacting via the particular interaction given above.
The gap equation is finally:

$$
\begin{aligned}
F(p)^{-1} & =-\lambda^{2} \int \frac{d^{d} q d^{d} k}{(2 \pi)^{2 d}} G(p-q-k) G(q) G(k), \\
G(p)^{-1} & =-3 \lambda^{2} \int \frac{d^{d} q d^{d} k}{(2 \pi)^{2 d}} G(p-q-k) F(q) G(k) .
\end{aligned}
$$

Dimensional analysis of the strong coupling fixed point actually does not fix $a$ : we get $b=-3 a+d$ from the first equation and $a=-2 a-b+d$ from the second equation. Let us try to solve the above equations, in the hope that numerical factors arising from the Feynman integrals may determine $a$. The overall constant $A$ is not determined from this procedure, but note that $[\lambda]=3-d$, and therefore $A \sim \lambda^{\frac{2(a-1)}{3-d}}$. This procedure is analogous to solving an eigenvalue equation, and perhaps it is not surprising that we have to do this, since a solution for $a$ also determines the anomalous dimension of a composite operator $\phi^{3}$. We then find

$$
F(p)=\frac{-1}{A^{3} \lambda^{2}} \frac{(2 \pi)^{2 d}}{L_{d}(a, a) L_{d}(2 a-d / 2, a)} \frac{1}{p^{2 b}},
$$

where

$L_{d}(a, b)=\pi^{d / 2} \frac{\Gamma(d / 2-a) \Gamma(d / 2-b) \Gamma(a+b-d / 2)}{\Gamma(a) \Gamma(b) \Gamma(d-a-b)}$.

The condition that must be satisfied by $a$ is then:

$$
3 \frac{L_{d}(2 a-d / 2, d-3 a)}{L_{d}(2 a-d / 2, a)}=1
$$

In position space, the IR two-point functions take the form

$$
G(x)=\frac{\Gamma(d / 2-a)}{\pi^{d / 2} 2^{2 a} \Gamma(a)} \frac{A}{\left(x^{2}\right)^{\Delta_{\phi}}},
$$

$F(x)=\frac{\Gamma(d / 2-b)}{\pi^{d / 2} 2^{2 b} \Gamma(b)} \frac{(2 \pi)^{2 d}}{A^{3} \lambda^{2} L_{d}(a, a) L_{d}(2 a-d / 2, a)} \frac{1}{\left(x^{2}\right)^{d-3 \Delta_{\phi}}}$.

In terms of $\Delta_{\phi},(2.9)$ may be written as

$$
f\left(d, \Delta_{\phi}\right) \equiv \frac{1}{3} \frac{\Gamma\left(\frac{d}{2}-3 \Delta_{\phi}\right) \Gamma\left(-\frac{d}{2}+3 \Delta_{\phi}\right) \Gamma\left(\Delta_{\phi}\right) \Gamma\left(d-\Delta_{\phi}\right)}{\Gamma\left(\frac{d}{2}-\Delta_{\phi}\right) \Gamma\left(-\frac{d}{2}+\Delta_{\phi}\right) \Gamma\left(3 \Delta_{\phi}\right) \Gamma\left(d-3 \Delta_{\phi}\right)}=1 .
$$

\section{A. The scaling dimension of $\phi$}

It can be verified numerically that solutions to (2.12) within the allowed range (2.5) do exist in $d<3$. 


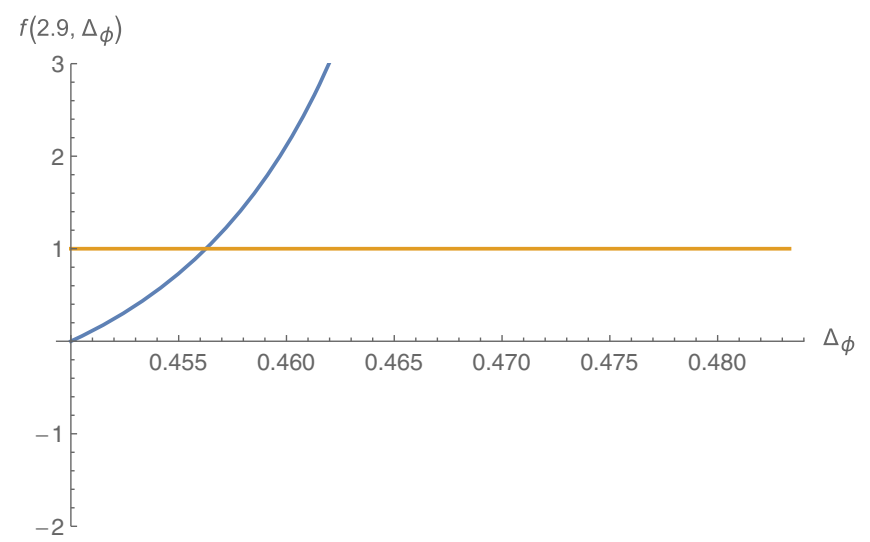

FIG. 3. Solving (2.12) for $d=2.9$.

For example, for $d=2.9$ we have the solution shown in Fig. 3:

$$
\Delta_{\phi} \approx 0.456, \quad \Delta_{\chi} \approx 1.531 .
$$

For $d=2.99$, we find $\Delta_{\phi}=0.495$, and $d=2.999$, $\Delta_{\phi}=0.4995$, consistent with the $3-\epsilon$ expansion (4.1). For $d=2,(2.9)$ simplifies to

$$
3\left(3 \Delta_{\phi}-1\right)^{2}=\left(\Delta_{\phi}-1\right)^{2} .
$$

The solution $\Delta_{\phi}=\frac{1}{13}(4-\sqrt{3})$ lies within the allowed range (2.5), while the one with the other branch of the square root is outside it.

For $d<2$ we find multiple solutions within the allowed range (2.5), as shown for $d=1$ in Fig. 4. One of the solutions gives $\Delta_{\phi}=0$; this produces singularities in the large $N$ dimensions of scalar bilinears, and we will not use it. The other solution,

$$
\Delta_{\phi} \approx-0.09055, \quad \Delta_{\chi} \approx 1.2717,
$$

appears to be acceptable. Although $\Delta_{\phi}$ is negative, it lies above the unitarity bound. We note that there is also a

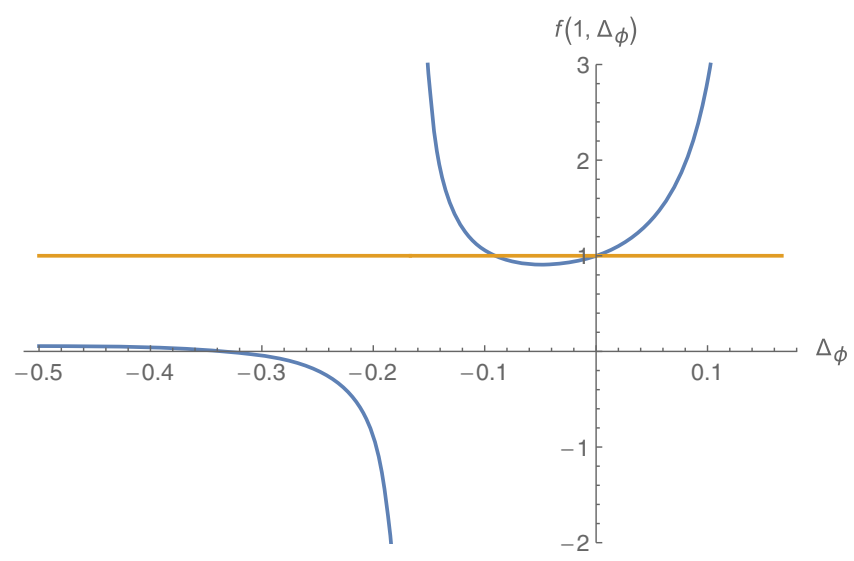

FIG. 4. Solving (2.12) for $d=1$. positive solution $\Delta_{\phi} \approx 0.225$, which lies outside of the allowed range (although it would be allowed if the $\chi$ field was dynamical).

There is an interesting transition in behavior which happens at $d=d_{c}$ where there is a double root at $\Delta_{\phi}=0$. The critical dimension $d_{c}$ is the solution of

$$
2+d_{c} \pi \cot \left(d_{c} \pi / 2\right)+d_{c}\left(\gamma+\psi\left(d_{c}\right)\right)=0 .
$$

Its numerical value is $d_{c}=1.35287$. For $d$ slightly above $d_{c}$ one of the solutions for $\Delta_{\phi}$ is zero, while the other is positive; we have to pick the positive one. However, for $d$ slightly below $d_{c}$ one of the solutions for $\Delta_{\phi}$ is zero, while the other is negative. Special care may be needed for continuation to $d<d_{c}$, in particular, for studying the $d=1$ case.

\section{BILINEAR OPERATORS}

There are three types of scalar bilinears one can consider, which are of the schematic form: $A=\phi(\xi \cdot \partial)^{s}\left(\partial^{2}\right)^{n} \phi, B=$ $\phi(\xi \cdot \partial)^{s}\left(\partial^{2}\right)^{n} \chi$ and $C=\chi(\xi \cdot \partial)^{s}\left(\partial^{2}\right)^{n} \chi$, where $\xi^{\mu}$ is an auxiliary null vector introduced to encode the spin of the operators, $\xi \cdot \partial=\xi^{\mu} \partial_{\mu}$, and $\partial^{2}=\partial^{\mu} \partial_{\mu}$. We note that there is mixing of operators of type $A$ and $C$. It is easy to convince oneself that there is no mixing with the $B$ operators by drawing a few diagrams.

\section{A. Bilinears of type B}

Let us consider a bilinear of type $B$, of spin $s$ and scaling dimension $\Delta$, for which there is no mixing. The three-point functions take the form $[26,27]$ :

$$
\begin{aligned}
& \left\langle\phi^{a b c}\left(x_{1}\right) \chi^{a b c}\left(x_{2}\right) B_{s}\left(x_{3} ; \xi\right)\right\rangle \\
& =v^{(B)}\left(x_{1}, x_{2}, x_{3}\right) \\
& =\frac{Q_{3}^{s}}{x_{31}^{\tau+\Delta_{\phi}-\Delta_{\chi}} x_{32}^{\tau+\Delta_{\chi}-\Delta_{\phi}} x_{12}^{\Delta_{\phi}+\Delta_{\chi}-\tau}} \rightarrow v_{s, \tau}^{(B)}\left(x_{1}, x_{2}\right) \\
& =\left(x_{12} \cdot \xi\right)^{s} x_{12}^{\tau-\Delta_{\phi}-\Delta_{\chi}},
\end{aligned}
$$

where $\tau=\Delta-s$ is the twist of the bilinear, $\xi$ is the null polarization vector, $Q_{3}$ is the conformally invariant tensor structure defined in $[26,27]$ and we took the limit $x_{3} \rightarrow \infty$ in the second line. The eigenvalue equation, obtained using the integration kernel depicted schematically in Fig. 5, is

$$
\begin{aligned}
v_{s, \tau}\left(x_{1}, x_{2}\right)= & 3 \lambda^{2} \int d^{d} y d^{d} z F\left(x_{2}, y\right) G(y, z)^{2} \\
& \times G\left(z, x_{1}\right) v_{s, \tau}(y, z)
\end{aligned}
$$

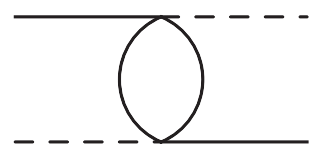

FIG. 5. The integration kernel for type B bilinears. 
When $s=0$, we have:

$$
\left|x_{1}-x_{2}\right|^{-\Delta_{\phi}-\Delta_{\chi}+\Delta}=3 \tilde{A}^{3} \tilde{B} \lambda^{2} \int d^{d} y d^{d} z \frac{1}{\left|x_{2}-y\right|^{2 \Delta_{\chi}}|y-z|^{5 \Delta_{\phi}+\Delta_{\chi}-\Delta}\left|z-x_{1}\right|^{2 \Delta_{\phi}}},
$$

which translates into

$$
g^{(B)}(d, \Delta) \equiv-3 \frac{\Gamma\left(3 \Delta_{\phi}\right) \sin \left(\frac{1}{2} \pi\left(d-6 \Delta_{\phi}\right)\right) \Gamma\left(\frac{d}{2}-\Delta_{\phi}\right) \Gamma\left(-\frac{d}{2}+3 \Delta_{\phi}+1\right) \Gamma\left(\frac{\Delta}{2}-\Delta_{\phi}\right) \Gamma\left(\frac{1}{2}\left(d-\Delta-2 \Delta_{\phi}\right)\right)}{\pi \Gamma\left(\Delta_{\phi}\right) \Gamma\left(\frac{\Delta}{2}+\Delta_{\phi}\right) \Gamma\left(\frac{d-\Delta}{2}+\Delta_{\phi}\right)}=1 .
$$

We can solve equation (3.4) numerically to find the allowed scaling dimensions for type B operators in various dimensions. In $d=2.9$ the type B scaling dimensions are

$$
\Delta_{B}=2.30120 ; 4.00173 ; 5.99214 ; 7.98983 ; 9.98891 ; \ldots,
$$

as shown in Fig. 6. In the pure $\phi$ language, the first one can be identified with the tetrahedral operator. The type B scaling approaches the asymptotic formula:

$$
\Delta_{B} \rightarrow 2 n+\Delta_{\phi}+\Delta_{\chi}=2 n+1.98747
$$

For example, for $n=54$ we numerically find $\Delta=$ 109.98749, which is very close to (3.6).

For spin $s>0$ the eigenvalue equation is:

$$
\begin{aligned}
& \left(x_{12} \cdot \xi\right)^{s}\left|x_{1}-x_{2}\right|^{-\Delta_{\phi}-\Delta_{\chi}+\Delta} \\
& =3 \tilde{A}^{3} \tilde{B} \lambda^{2} \int d^{d} y d^{d} z \frac{((y-z) \cdot \xi)^{s}}{\left|x_{2}-y\right|^{2 \Delta_{\chi}}|y-z|^{5 \Delta_{\phi}+\Delta_{\chi}-\Delta}\left|z-x_{1}\right|^{2 \Delta_{\phi}}} .
\end{aligned}
$$

Note that the spectrum of type $B$ bilinears does not contain the stress tensor, which is of type $A / C$.

Processing the equation we have the following condition for the allowed twists of higher spin bilinears:

$$
g^{(B)}(d, \tau, s) \equiv-3 \frac{\Gamma\left(3 \Delta_{\phi}\right) \sin \left(\frac{1}{2} \pi\left(d-6 \Delta_{\phi}\right)\right) \Gamma\left(\frac{d}{2}-\Delta_{\phi}\right) \Gamma\left(-\frac{d}{2}+3 \Delta_{\phi}+1\right) \Gamma\left(\frac{1}{2}\left(d-2 \Delta_{\phi}-\tau\right)\right) \Gamma\left(s-\Delta_{\phi}+\frac{\tau}{2}\right)}{\pi \Gamma\left(\Delta_{\phi}\right) \Gamma\left(\frac{d}{2}+\Delta_{\phi}-\frac{\tau}{2}\right) \Gamma\left(s+\Delta_{\phi}+\frac{\tau}{2}\right)}=1 .
$$

Using this equation one can find the allowed twists of spin- $s$ type B bilinears. For example, the spectrum when $s=2$ and $d=2.9$ is found from Fig. 7 to be $\tau=2.08$, $3.99,5.99,7.99, \ldots$, which approach $\Delta_{\chi}+\Delta_{\phi}+2 n=$ $1.99+2 n$ from above.

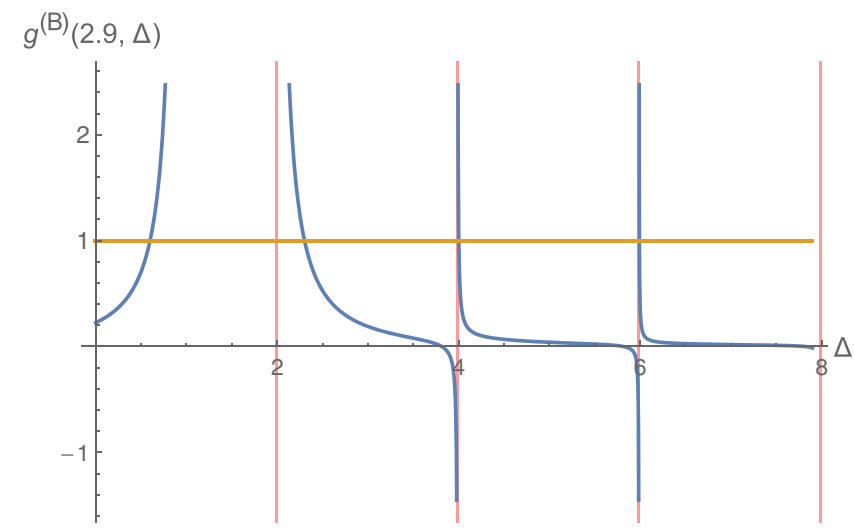

FIG. 6. The spectrum of type B bilinears in $d=2.9$. The red lines correspond to asymptotes at $2 n+\Delta_{\phi}+\Delta_{\chi}=$ $2 n+1.98747$.
We find that the spectrum of type B bilinears appears to be real for all $d<3$. However, there are ranges of $d$ where the spectrum of type $\mathrm{A} / \mathrm{C}$ operators do contain complex eigenvalues, as we discuss in the next section.

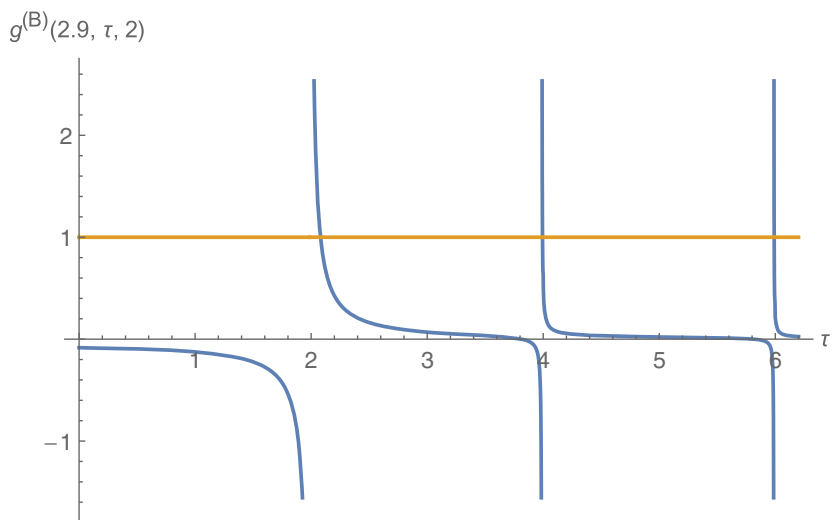

FIG. 7. Solving equation (3.8) in $d=2.9$ for the allowed twists of spin-2 type $B$ bilinears. 


\section{B. Mixing of bilinears of type A and C}

Let us now study the spectrum of bilinear operators of type $A$ and $C$. As mentioned earlier, by drawing a few diagrams (see Fig. 8) one can see that these operators mix, in the sense that the two-point function $\left\langle A_{s} C_{s}\right\rangle \neq 0$. Let $\tau=\Delta-s$ be the twist of mixture of $A$ and $C$ operators, which we denote as $\tilde{A}_{s}$. As in the previous subsection, from the three-point functions $\left\langle\phi^{a b c}\left(x_{1}\right) \phi^{a b c}\left(x_{2}\right) \tilde{A}_{s}\left(x_{3} ; \xi\right)\right\rangle$ and $\left\langle\chi^{a b c}\left(x_{1}\right) \chi^{a b c}\left(x_{2}\right) \tilde{A}_{s}\left(x_{3} ; \xi\right)\right\rangle$, we define

$v_{s, \tau}^{(A)}(x, y)=\frac{((x-y) \cdot \xi)^{s}}{(x-y)^{2 \Delta_{\phi}-\tau}}, \quad v_{s, \tau}^{(C)}(x, y)=\frac{((x-y) \cdot \xi)^{s}}{(x-y)^{2 \Delta_{x}-\tau}}$.

We now define the following kernels, depicted schematically in Fig. 8:

$$
\begin{aligned}
K_{A A}\left[v^{(A)}\right]= & 3 \int d^{d} x d^{d} y G\left(x_{1}, x\right) G\left(x_{2}, y\right) G(x, y) \\
& F(x, y) v_{s, \tau}^{(A)}(x, y) \\
K_{C A}\left[v^{(A)}\right]= & 3 \int d^{d} x d^{d} y F\left(x_{1}, x\right) F\left(x_{2}, y\right) G(x, y)^{2} v_{s, \tau}^{(A)}(x, y)
\end{aligned}
$$

$$
K_{A C}\left[v^{(C)}\right]=3 \int d^{d} x d^{d} y G\left(x_{1}, x\right) G\left(x_{2}, y\right) G(x, y)^{2} v_{s, \tau}^{(C)}(x, y) .
$$

Note the factor of 3, which appears from a careful counting of the Wick contractions.

The integration kernel gives rise to the following matrix:

$$
\left(\begin{array}{cc}
2 K_{A A}\left[v^{(A)}\right] / v^{(A)} & K_{A C}\left[v^{(C)}\right] / v^{(A)} \\
K_{C A}\left[v^{(A)}\right] / v^{(C)} & 0
\end{array}\right) \equiv\left(\begin{array}{cc}
2 K_{1} & K_{3} \\
K_{2} & 0
\end{array}\right) .
$$

The condition for it to have eigenvalue 1 , which determines the allowed values of $\tau$, is

$$
g^{(A)}(d, \tau, s) \equiv 2 K_{1}+K_{3} K_{2}=1 .
$$

Luckily, this condition is independent of the constant $A$, as one can see from the following expressions,

$$
\begin{aligned}
K_{1} & =\frac{3\left(d-6 \Delta_{\phi}\right) \Gamma\left(3 \Delta_{\phi}\right) \sin \left(\frac{1}{2} \pi\left(d-6 \Delta_{\phi}\right)\right) \Gamma\left(d-3 \Delta_{\phi}\right) \Gamma\left(\frac{d}{2}-\Delta_{\phi}\right)^{2} \Gamma\left(\Delta_{\phi}-\frac{\tau}{2}\right) \Gamma\left(-\frac{d}{2}+s+\Delta_{\phi}+\frac{\tau}{2}\right)}{2 \pi \Gamma\left(\Delta_{\phi}\right)^{2} \Gamma\left(d-\Delta_{\phi}-\frac{\tau}{2}\right) \Gamma\left(\frac{1}{2}\left(d+2 s-2 \Delta_{\phi}+\tau\right)\right)}, \\
K_{2} & =\frac{3 \pi^{d} 2^{4\left(d-2 \Delta_{\phi}\right)} \Gamma\left(3 \Delta_{\phi}\right)^{2} \Gamma\left(\frac{d}{2}-\Delta_{\phi}\right)^{4} \Gamma\left(d-3 \Delta_{\phi}-\frac{\tau}{2}\right) \Gamma\left(\frac{1}{2}\left(d+2 s-6 \Delta_{\phi}+\tau\right)\right)}{A^{4} \lambda^{2} \Gamma\left(\Delta_{\phi}\right)^{4} \Gamma\left(\frac{d}{2}-3 \Delta_{\phi}\right)^{2} \Gamma\left(3 \Delta_{\phi}-\frac{\tau}{2}\right) \Gamma\left(-\frac{d}{2}+s+3 \Delta_{\phi}+\frac{\tau}{2}\right)}, \\
K_{3} & =\frac{3 A^{4} \pi^{-d} \lambda^{2} 2^{8 \Delta_{\phi}-4 d} \Gamma\left(\Delta_{\phi}\right)^{2} \Gamma\left(\Delta_{\phi}-\frac{\tau}{2}\right) \Gamma\left(-\frac{d}{2}+s+\Delta_{\phi}+\frac{\tau}{2}\right)}{\Gamma\left(\frac{d}{2}-\Delta_{\phi}\right)^{2} \Gamma\left(d-\Delta_{\phi}-\frac{\tau}{2}\right) \Gamma\left(\frac{1}{2}\left(d+2 s-2 \Delta_{\phi}+\tau\right)\right)} .
\end{aligned}
$$

Thus, the equation we need to solve is:

$$
\begin{gathered}
\frac{\Gamma\left(\Delta_{\phi}\right)^{2} \Gamma\left(\frac{d}{2}-3 \Delta_{\phi}\right)^{2} \Gamma\left(3 \Delta_{\phi}-\frac{d}{2}\right) \Gamma\left(3 \Delta_{\phi}-\frac{\tau}{2}\right) \Gamma\left(d-\Delta_{\phi}-\frac{\tau}{2}\right) \Gamma\left(-\frac{d}{2}+s+3 \Delta_{\phi}+\frac{\tau}{2}\right) \Gamma\left(\frac{1}{2}\left(d+2 s-2 \Delta_{\phi}+\tau\right)\right)}{3 \Gamma\left(3 \Delta_{\phi}\right) \Gamma\left(\frac{d}{2}-\Delta_{\phi}\right)^{2} \Gamma\left(\Delta_{\phi}-\frac{\tau}{2}\right) \Gamma\left(-\frac{d}{2}+s+\Delta_{\phi}+\frac{\tau}{2}\right)} \\
=3 \Gamma\left(3 \Delta_{\phi}\right) \Gamma\left(3 \Delta_{\phi}-\frac{d}{2}\right) \Gamma\left(d-3 \Delta_{\phi}-\frac{\tau}{2}\right) \Gamma\left(\frac{1}{2}\left(d+2 s-6 \Delta_{\phi}+\tau\right)\right) \\
-2 \Gamma\left(\frac{d}{2}-3 \Delta_{\phi}\right) \Gamma\left(d-3 \Delta_{\phi}\right) \Gamma\left(3 \Delta_{\phi}-\frac{\tau}{2}\right) \Gamma\left(-\frac{d}{2}+s+3 \Delta_{\phi}+\frac{\tau}{2}\right) .
\end{gathered}
$$
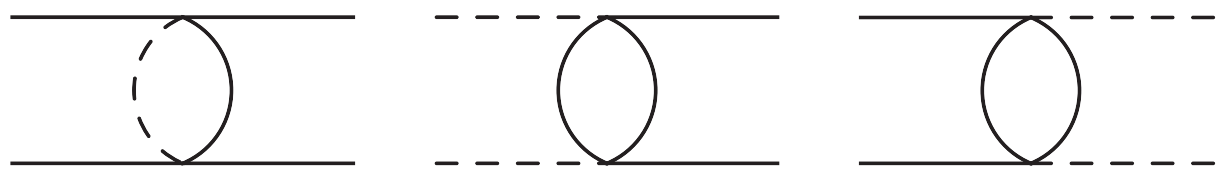

FIG. 8. The integration kernels $K_{A A}, K_{C A}$ and $K_{A C}$, respectively, for mixtures of type $A$ and $C$ bilinears. 


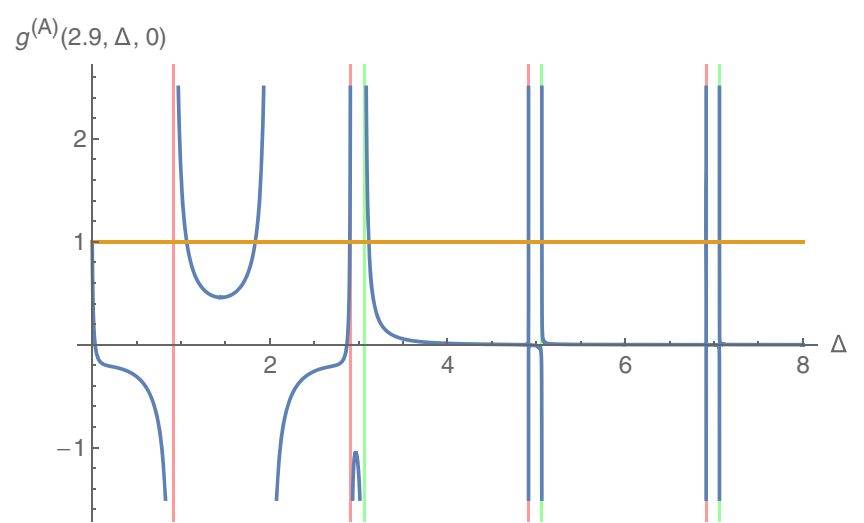

FIG. 9. The spectrum of type A/C scalar bilinears in $d=2.9$. The green lines correspond to the $2 \Delta_{\chi}+2 n$ asymptotics and the red ones to $2 \Delta_{\phi}+2 n$ asymptotics. We see that the solutions are real and approach the expected values as $n \rightarrow \infty$.

One can check that the stress-tensor, which has $s=2$ and $\tau=d-2$, appears in this spectrum for any $d$.

The Schwinger-Dyson equations have a symmetry under $\Delta \rightarrow d-\Delta$. In a given conformal field theory (CFT), only one of this pair of solutions corresponds to a primary operator dimension, while the other one is its "shadow." The $s=0$ spectrum contains complex modes for $1.6799<d<2.8056$. In $d=2.9$ the graphical solution for the scaling dimensions in the type $\mathrm{A} / \mathrm{C}$ sector is shown in Fig. 9. The lowest few are

$\Delta=1.064,1.836,2.9,3.114,4.912,5.063,6.913,7.063, \ldots$

The eigenvalue at $\Delta=2.9$ is exact, and in general $\Delta=d$ is an eigenvalue for any $d$. The solution 1.836 corresponds to the shadow of 1.064. As $d$ is further lowered, the part of the graph between 1 and 2 moves up so that the two solutions become closer. In $d=d_{\text {crit }}$, where $d_{\text {crit }} \approx 2.8056$, the two

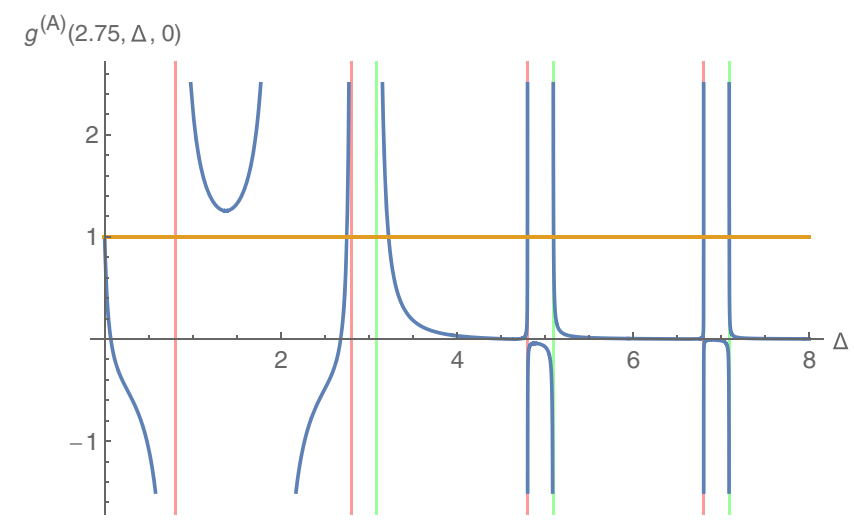

FIG. 10. The spectrum of type A/C scalar bilinears in $d=2.75$. The green lines correspond to the $2 \Delta_{\chi}+2 n$ asymptotics and the red ones to $2 \Delta_{\phi}+2 n$ asymptotics. We see that two real solutions are no longer present; they are now complex.

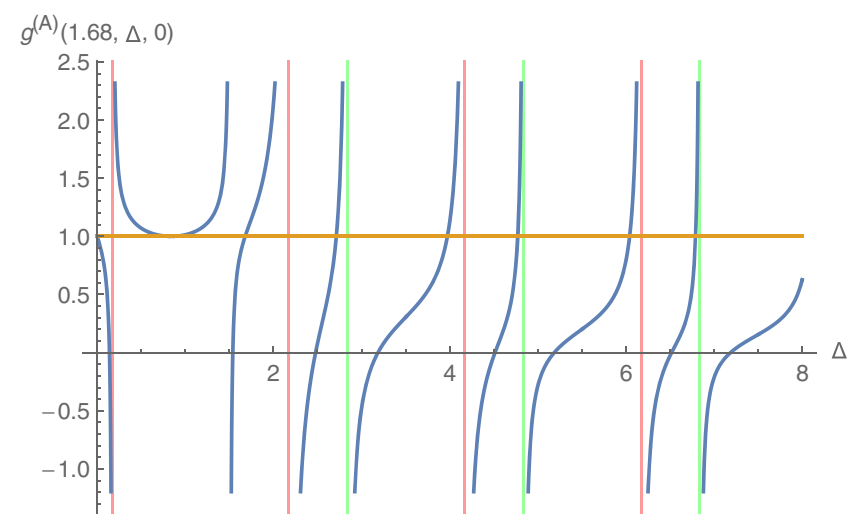

FIG. 11. The spectrum of type A/C scalar bilinears in $d=1.68$. The green vertical lines correspond to the $2 \Delta_{\chi}+2 n$ asymptotics, the red ones to the $2 \Delta_{\phi}+2 n$ asymptotics.

solutions merge into a single one at $d / 2$ (for discussions of mergers of fixed points, see [28-30]). For $d<d_{\text {crit }}$, the solutions become complex $\frac{d}{2} \pm i \alpha(d)$ and the prismatic model becomes unstable. The plot for $d=2.75$ is shown in Fig. 10.

For $d \leq 1.68$, the spectrum of bilinears is again real. The plot for $d=1.68$, where $\Delta_{\phi} \approx 0.0867$, is shown in Fig. 11 . At this critical value of $d$ there are two solutions at $d / 2$; one is the shadow of the other.

\section{LARGE $\boldsymbol{N}$ RESULTS IN $3-\epsilon$ DIMENSIONS}

Let us solve the Schwinger-Dyson equations in $d=3-\epsilon$. The results will be compared with renormalized perturbation theory in the following section. The scaling dimension of $\phi^{a b c}$ is found to be

$$
\begin{aligned}
\Delta_{\phi}= & \frac{1}{2}-\frac{\epsilon}{2}+\epsilon^{2}-\frac{20 \epsilon^{3}}{3}+\left(\frac{472}{9}+\frac{\pi^{2}}{3}\right) \epsilon^{4} \\
& +\left(7 \zeta(3)-\frac{12692}{27}-\frac{56 \pi^{2}}{9}\right) \epsilon^{5}+\mathcal{O}\left(\epsilon^{6}\right)
\end{aligned}
$$

This is within the allowed range (2.5) and is close to its upper boundary. The scaling dimension of $\chi^{a b c}$ is

$$
\begin{aligned}
\Delta_{\chi}= & d-3 \Delta_{\phi}=\frac{3}{2}+\frac{\epsilon}{2}-3 \epsilon^{2}+20 \epsilon^{3}-\left(\frac{472}{3}+\pi^{2}\right) \epsilon^{4} \\
& -3\left(7 \zeta(3)-\frac{12692}{27}-\frac{56 \pi^{2}}{9}\right) \epsilon^{5}+\mathcal{O}\left(\epsilon^{6}\right) .
\end{aligned}
$$

Let us consider the $s=0$ type A/C bilinears. For the first eigenvalue we find

$$
\begin{aligned}
\Delta_{\phi^{2}}= & 1-\epsilon+32 \epsilon^{2}-\frac{976 \epsilon^{3}}{3}+\left(\frac{30320}{9}+\frac{32 \pi^{2}}{3}\right) \epsilon^{4} \\
& +\mathcal{O}\left(\epsilon^{5}\right) .
\end{aligned}
$$


It corresponds to the scaling dimension of operator $\phi^{a b c} \phi^{a b c}$, as we will show in the next section. The next eigenvalue is the shadow dimension $d-\Delta_{\phi^{2}}$.

The next solution of the Schwinger-Dyson equation is $\Delta=d=3-\epsilon$ for all $d$. While this seems to correspond to an exactly marginal operator, we believe that the corresponding operator is redundant: it is a linear combination of $\phi^{a b c} \partial^{2} \phi^{a b c}$ and $\chi^{a b c} \chi^{a b c}$. Similar redundant operators with $h=1$ showed up in the Schwinger-Dyson analysis of multiflavor models [12,31]. They decouple in correlation functions [12] and were shown to vanish by the equations of motion [31]. The next eigenvalue is

$$
\begin{aligned}
\Delta_{\text {prism }}= & 3+\epsilon+6 \epsilon^{2}-84 \epsilon^{3}+\left(\frac{1532}{3}+10 \pi^{2}\right) \epsilon^{4} \\
& +\left(18 \zeta(3)-\frac{6392}{3}-\frac{452 \pi^{2}}{3}\right) \epsilon^{5}+\mathcal{O}\left(\epsilon^{6}\right) .
\end{aligned}
$$

It should correspond to the sextic prism operator (1.2), which is related by the equations of motion to a linear combination of $\phi^{a b c} \partial^{2} \phi^{a b c}$ and $\chi^{a b c} \chi^{a b c}$.

The subsequent eigenvalues may be separated into two sets. One of them has the form, for integer $n \geq 0$,

$$
\Delta_{n}^{-}=5+2 n-\epsilon+2 \epsilon^{2}-\frac{40 \epsilon^{3}}{3}+\frac{\left(2\left(472+3 \pi^{2}\right) n(2 n+7)(n(2 n+7)+11)+180 \pi^{2}+28212\right) \epsilon^{4}}{9(n+1)(n+2)(2 n+3)(2 n+5)}+\mathcal{O}\left(\epsilon^{5}\right) .
$$

For large $n$ this approaches $4+2 n+2 \Delta_{\phi}$, as expected for an operator of the form $\phi^{a b c}\left(\partial^{2}\right)^{2+n} \phi^{a b c}$. The other set of eigenvalues has the form, for integer $n \geq 0$,

$$
\begin{aligned}
\Delta_{n}^{+}= & 5+2 n+\epsilon-6 \epsilon^{2}+4\left(\frac{9}{n+2}-\frac{18}{2 n+3}\right. \\
& \left.-\frac{6}{2 n+5}+\frac{3}{n+1}+10\right) \epsilon^{3}+\mathcal{O}\left(\epsilon^{4}\right) .
\end{aligned}
$$

For large $n$ this approaches $2+2 n+2 \Delta_{\chi}$, as expected for an operator of the form $\chi^{a b c}\left(\partial^{2}\right)^{1+n} \chi^{a b c}$. These simple asymptotic forms suggest that for large $n$ the mixing between operators $\phi^{a b c}\left(\partial^{2}\right)^{2+n} \phi^{a b c}$ and $\chi^{a b c}\left(\partial^{2}\right)^{1+n} \chi^{a b c}$ approaches zero.

We can also use (3.4) to derive the $3-\epsilon$ expansions of the dimensions of type B operators,

$$
O_{B, n}=\chi^{a b c}\left(\partial_{\mu} \partial^{\mu}\right)^{n} \phi^{a b c}+\ldots,
$$

where the additional terms are there to make them conformal primaries. For $n=0$ we find

$\Delta_{B, 0}=2+6 \epsilon-68 \epsilon^{2}+\frac{2848+24 \pi^{2}}{3} \epsilon^{3}+\mathcal{O}\left(\epsilon^{4}\right)$.

This scaling dimension corresponds to the operator $\phi^{a b c} \chi^{a b c}$, which in the original $\phi$ language is the tetrahedron operator $O_{\text {tetra. }}$. For the higher operators we get

$$
\Delta_{B, 1}=4+4 \epsilon^{3}-44 \epsilon^{4}+\mathcal{O}\left(\epsilon^{5}\right),
$$

$$
\Delta_{B, 2}=6-\frac{7}{5} \epsilon^{2}+\frac{331}{30} \epsilon^{3}-\left(\frac{199547}{2250}+\frac{7 \pi^{2}}{15}\right) \epsilon^{4}+\mathcal{O}\left(\epsilon^{5}\right),
$$

$$
\begin{aligned}
\Delta_{B, 3}= & 8-\frac{12}{7} \epsilon^{2}+\frac{9139}{735} \epsilon^{3}-\left(\frac{7581556}{77175}+\frac{4 \pi^{2}}{7}\right) \epsilon^{4} \\
& +\mathcal{O}\left(\epsilon^{5}\right), \text { etc. }
\end{aligned}
$$

Using the equations of motion, we can write $O_{B, 1}$, up to a total derivative, as a sum of the three 8-particle operators shown in the leftmost column of Fig. 9 in [31]. In general, for $n>0$,

$\Delta_{B, n}=2 n+2-2\left(1-\frac{3}{n(2 n+1)}\right) \epsilon^{2}+\mathcal{O}\left(\epsilon^{3}\right)$,

which agrees for large $n$ with the expected asymptotic behavior

$\Delta_{B, n} \rightarrow 2 n+\Delta_{\phi}+\Delta_{\chi}=2 n+2-2 \epsilon^{2}+\mathcal{O}\left(\epsilon^{3}\right)$.

\section{A. Higher spin spectrum}

Let us also present the $\epsilon$ expansions for the higher spin bilinear operators which are mixtures of type $A$ and $C$. The lowest eigenvalue of twist $\tau=\Delta-s$ for spin $s$ is

$\tau_{0}=1-\epsilon+\frac{8\left(s^{2}-4\right) \epsilon^{2}}{4 s^{2}-1}+\frac{4 \epsilon^{3}\left(27\left(1-4 s^{2}\right) H_{s-\frac{1}{2}}-2 s\left(80 s^{3}+s(54 \log (4)-508)+45\right)-244+27 \log (4)\right)}{3\left(1-4 s^{2}\right)^{2}}+\mathcal{O}\left(\epsilon^{4}\right)$, 
where $H_{n}$ is the harmonic number and the last two terms (as well as all higher-order terms) vanish when $s=2$ as expected. In the large $s$ limit, this becomes:

$$
\begin{aligned}
\tau_{0} & \rightarrow 1-\epsilon+\epsilon^{2}\left(2-\frac{15}{2 s^{2}}+\mathcal{O}\left(s^{-3}\right)\right) \\
& +\epsilon^{3}\left(-\frac{40}{3}+\frac{-9 \log (4 s)-9 \gamma+78}{s^{2}}+\mathcal{O}\left(s^{-3}\right)\right) \\
& +\mathcal{O}\left(\epsilon^{4}\right) .
\end{aligned}
$$

Comparing with (5.8), we see that

$$
\tau_{0}=2 \Delta_{\phi}+O\left(\frac{1}{s^{2}}\right)
$$

This is the expected large spin limit [32-35] for an operator bilinear in $\phi$, indicating that for large spin the mixing with $\chi$ bilinears is suppressed.

The next two twists are

$$
\begin{aligned}
\tau_{1}= & 3-\epsilon+\frac{8 s(s+2) \epsilon^{2}}{4 s(s+2)+3}+\frac{4 \epsilon^{3}}{3(4 s(s+2)+3)^{2}}\left(-4(40 s(s+4)+157) s^{2}+6(s+27)-27 \gamma(4 s(s+2)+3)\right. \\
& \left.-27(4 s(s+2)+3) \log (4)-27(4 s(s+2)+3) \psi\left(s+\frac{3}{2}\right)\right)+\mathcal{O}\left(\epsilon^{4}\right),
\end{aligned}
$$

and

$$
\begin{aligned}
\tau_{2}= & 3+\epsilon+\left(\frac{36}{4 s(s+2)+3}-6\right) \epsilon^{2}+\frac{4 \epsilon^{3}}{(4 s(s+2)+3)^{2}}(4 s(2 s(20 s(s+4)+56+9 \log 4)-105+36 \log 4) \\
& \left.+18 \gamma(4 s(s+2)+3)+18(4 s(s+2)+3) \psi\left(s+\frac{3}{2}\right)-297+54 \log 4\right)+\mathcal{O}\left(\epsilon^{4}\right),
\end{aligned}
$$

where $\psi(x)$ is the digamma function. In the large $s$ limit, these take the form,

$$
\begin{aligned}
& \tau_{1} \rightarrow 3-\epsilon+\epsilon^{2}\left(2-\frac{3}{2 s^{2}}+O\left(s^{-3}\right)\right)+\epsilon^{3}\left(-\frac{40}{3}-\frac{3(3 \log (s)+\log (64)+3 \gamma-7)}{s^{2}}+O\left(s^{-3}\right)\right)+O\left(\epsilon^{4}\right) \\
& =2 \Delta_{\phi}+2+O\left(\frac{1}{s^{2}}\right)
\end{aligned}
$$

and

$$
\begin{aligned}
& \tau_{2} \rightarrow 3+\epsilon+\epsilon^{2}\left(-6+\frac{9}{s^{2}}+O\left(s^{-3}\right)\right)+\epsilon^{3}\left(40+\frac{18(\log (s)+\log (4)+\gamma-6)}{s^{2}}+O\left(s^{-3}\right)\right)+O\left(\epsilon^{4}\right) \\
& =2 \Delta_{\chi}+O\left(\frac{1}{s^{2}}\right)
\end{aligned}
$$

In general, for large spin we find the two towers of twists labeled by an integer $n$,

$$
\begin{aligned}
& \tau_{n}^{A}=2 n+1-\epsilon+2 \epsilon^{2}-\frac{40 \epsilon^{3}}{3}+\mathcal{O}\left(\epsilon^{4}\right)=2 \Delta_{\phi}+2 n+\ldots \\
& \tau_{n}^{C}=2 n+3+\epsilon-6 \epsilon^{2}+\mathcal{O}\left(\epsilon^{3}\right)=2 \Delta_{\chi}+2 n+\ldots
\end{aligned}
$$

again in agreement with the expected asymptotics and suppression of mixing at large spin.

We can similarly derive explicit results for spinning operators in the type B sector using (3.8). For the lowest two twists, we find 


$$
\begin{aligned}
\tau_{0} & =2+\frac{6 \epsilon}{2 s+1}+\frac{2 \epsilon^{2}\left(3(2 s+1)^{2}\left(H_{s-\frac{1}{2}}+\log (4)\right)-8 s^{3}-84 s^{2}-72 s-34\right)}{(2 s+1)^{3}}+O\left(\epsilon^{3}\right) \\
& =\left(2-2 \epsilon^{2}+O\left(\epsilon^{3}\right)\right)+O\left(\frac{1}{s}\right), \\
\tau_{1} & =4-\frac{4 s \epsilon^{2}}{2 s+3}+\frac{2 \epsilon^{3}\left(9(2 s+3) H_{s+\frac{1}{2}}+80 s^{2}+12 s(8+\log (8))+54 \log (2)\right)}{3(2 s+3)^{2}}+O\left(\epsilon^{4}\right) \\
& =\left(4-2 \epsilon^{2}+\frac{40 \epsilon^{3}}{3}+O\left(\epsilon^{4}\right)\right)+O\left(\frac{1}{s}\right)
\end{aligned}
$$

and higher twists may be analyzed similarly. One can see that these results are also in agreement with the expected large spin limit $\tau_{n} \rightarrow \Delta_{\phi}+\Delta_{\chi}+2 n$ for fixed $n$.

\section{RENORMALIZED PERTURBATION THEORY}

In this section we use the renormalized perturbation theory to carry out the $3-\epsilon$ expansion for finite $N$. We will find a fixed point with real couplings, whose large $N$ limit reproduces the results found using the $3-\epsilon$ expansion of the Schwinger-Dyson solution in the previous section. This is an excellent check of the Schwinger-Dyson approach to the prismatic theory.

To carry out the beta function calculation at finite $N$ we need to include all the $O(N)^{3}$ invariant sextic terms in the action (as usual in such calculations, we ignore the quartic and quadratic operators which are relevant in $d=3$ ). The 11 such single-sum terms are shown diagrammatically in Fig. 5 of [31]. We will impose the additional constraint that the action is invariant under the permutation group $S_{3}$ which acts on the three $O(N)$ symmetry groups. This leaves us with 8 operators: 5 single-sum, 2 double-sum and 1 triple-sum. They are written down explicitly in (A1) and shown schematically in Fig. 1. The first one, and the most essential one for achieving the solvable large $N$ limit, is the "prism" term (1.2); it is positive definite and symmetric under the interchanges of the three $O(N)$ groups.

Our action is a special case of a general multifield $\phi^{6}$ tensor theory:

$$
\begin{aligned}
S= & \int d^{d} x\left(\frac{1}{2} \partial_{\mu} \phi^{a b c} \partial^{\mu} \phi^{a b c}\right. \\
& \left.+\frac{1}{6 !} g_{\kappa_{1} \kappa_{2} \kappa_{3} \kappa_{4} \kappa_{5} \kappa_{6}} \phi^{\kappa_{1}} \phi^{\kappa_{2}} \phi^{\kappa_{3}} \phi^{\kappa_{4}} \phi^{\kappa_{5}} \phi^{\kappa_{6}}\right) .
\end{aligned}
$$

The beta functions and anomalous dimensions for such a general sextic coupling were calculated in [36,37]; see also [38,39] for earlier results on the $O(n)$ invariant sextic theory. The diagram topology contributing to the leading two-loop beta function is shown in Fig. 12 .
In our case each index $\kappa_{1}, \kappa_{2} \ldots, \kappa_{6}$ has three sub indices $\kappa_{i}=\left(a_{i} b_{i} c_{i}\right)$. The coupling $g_{\kappa_{1} \kappa_{2} \kappa_{3} \kappa_{4} \kappa_{5} \kappa_{6}}$ contains 8 different types of interactions:

$$
\begin{aligned}
g_{\kappa_{1} \kappa_{2} \kappa_{3} \kappa_{4} \kappa_{5} \kappa_{6}}= & g_{1} T_{\kappa_{1} \kappa_{2} \kappa_{3} \kappa_{4} \kappa_{5} \kappa_{6}}^{(1)}+g_{2} T_{\kappa_{1} \kappa_{2} \kappa_{3} \kappa_{4} \kappa_{5} \kappa_{6}}^{(2)} \\
& +\cdots+g_{8} T_{\kappa_{1} \kappa_{2} \kappa_{3} \kappa_{4} \kappa_{5} \kappa_{6}}^{(8)},
\end{aligned}
$$

which can be graphically represented as in Fig. 1. Each tensor structure $T_{\kappa_{1} \kappa_{2} \kappa_{3} \kappa_{4} \kappa_{5} \kappa_{6}}^{(k)}$ consists of a sum of product of $\delta$ functions, which are symmetrized over the colors $(a b c)$ and over the indices $\kappa_{1}, \ldots, \kappa_{6}$.

The two-loop beta functions and anomalous dimensions for general $N$ are given in the Appendix. Let us use the large $N$ scaling

$$
\begin{aligned}
g_{1} & =180 \cdot(8 \pi)^{2} \epsilon \frac{\tilde{g}_{1}}{N^{3}}, \quad g_{2,4,6,7}=180 \cdot(8 \pi)^{2} \epsilon \frac{\tilde{g}_{2,4,6,7}}{N^{5}}, \\
g_{3,5} & =180 \cdot(8 \pi)^{2} \epsilon \frac{\tilde{g}_{3,5}}{N^{4}}, \quad g_{8}=180 \cdot(8 \pi)^{2} \epsilon \frac{\tilde{g}_{8}}{N^{7}},
\end{aligned}
$$

which is chosen in such a way that all beta functions retain nonvanishing quadratic terms in the large $N$ limit:

$$
\begin{aligned}
\tilde{\beta}_{1}= & -2 \tilde{g}_{1}+2 \tilde{g}_{1}^{2}, \\
\tilde{\beta}_{2}= & -2 \tilde{g}_{2}+4 \tilde{g}_{1}\left(3 \tilde{g}_{1}+2 \tilde{g}_{5}\right), \\
\tilde{\beta}_{3}= & -2 \tilde{g}_{3}+12 \tilde{g}_{1}^{2}, \\
\tilde{\beta}_{4}= & -2 \tilde{g}_{4}+\frac{2}{3}\left(2\left(3 \tilde{g}_{1}+\tilde{g}_{3}\right)^{2}+\tilde{g}_{5}^{2}+12 \tilde{g}_{1} \tilde{g}_{5}\right), \\
\tilde{\beta}_{5}= & -2 \tilde{g}_{5}+4 \tilde{g}_{1}\left(6 \tilde{g}_{1}+\tilde{g}_{5}\right), \\
\tilde{\beta}_{6}= & -2 \tilde{g}_{6}+4 \tilde{g}_{1}\left(3 \tilde{g}_{1}+\tilde{g}_{5}+2 \tilde{g}_{6}\right), \\
\tilde{\beta}_{7}= & -2 \tilde{g}_{7}+6 \tilde{g}_{1}^{2}, \\
\tilde{\beta}_{8}= & -2 \tilde{g}_{8}+\frac{4}{3}\left(\tilde{g}_{3}^{2}+4 \tilde{g}_{7} \tilde{g}_{3}+\tilde{g}_{5}^{2}+6 \tilde{g}_{6}^{2}+2 \tilde{g}_{7}^{2}\right. \\
& \left.+6 \tilde{g}_{5} \tilde{g}_{6}+3 \tilde{g}_{1}\left(\tilde{g}_{5}+6 \tilde{g}_{6}\right)\right) .
\end{aligned}
$$




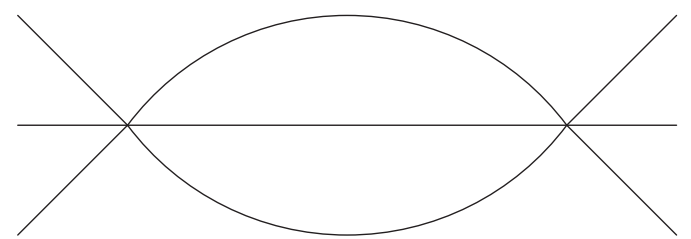

FIG. 12. The two-loop contribution to the beta function.

The unique nontrivial fixed point of these scaled beta functions is at

$\begin{array}{lrll}\tilde{g}_{1}^{*}=1, & \tilde{g}_{2}^{*}=-42, & \tilde{g}_{3}^{*}=6, & \tilde{g}_{4}^{*}=54, \\ \tilde{g}_{5}^{*}=-12, & \tilde{g}_{6}^{*}=6, & \tilde{g}_{7}^{*}=3, & \tilde{g}_{8}^{*}=84 .\end{array}$

For this fixed point, the eigenvalues of the matrix $\frac{\partial \tilde{\beta}_{i}}{\partial \tilde{g}_{j}}$ are

$\lambda_{i}=6, \quad 2, \quad 2, \quad-2, \quad-2, \quad-2, \quad-2, \quad-2$.

That there are unstable directions at the "prismatic" fixed point also follows from the solution of the Schwinger-Dyson equations. ${ }^{3}$ Using (4.3) we see that the large $N$ dimension of the triple-trace operator $\left(\phi^{a b c} \phi^{a b c}\right)^{3}$ is $3(1-\epsilon)+\mathcal{O}\left(\epsilon^{2}\right)$, which means that it is relevant in $d=3-\epsilon$ and is one of the operators corresponding to eigenvalue -2 . On the other hand, the prism operator is irrelevant and corresponds to eigenvalue 2. Another irrelevant operator is $O_{\text {tetra }} \phi^{a b c} \phi^{a b c}$; from (4.8) it follows that its large $N$ dimension is $3+5 \epsilon+\mathcal{O}\left(\epsilon^{2}\right)$, so it corresponds to eigenvalue 6 .

We have also calculated the $1 / N$ corrections to the fixed point (5.5):

$$
\begin{aligned}
& \tilde{g}_{1}^{*}=1-\frac{6}{N}+\frac{18}{N^{2}}+\ldots, \\
& \tilde{g}_{2}^{*}=-42+\frac{384}{N}+\frac{8592}{N^{2}}+\ldots, \\
& \tilde{g}_{3}^{*}=6+\frac{1848}{N^{2}}+\ldots, \\
& \tilde{g}_{4}^{*}=54-\frac{132}{N}+\frac{16392}{N^{2}}+\ldots, \\
& \tilde{g}_{5}^{*}=-12+\frac{30}{N}+\frac{2340}{N^{2}}+\ldots, \\
& \tilde{g}_{6}^{*}=6+\frac{36}{N}-\frac{1320}{N^{2}}+\ldots, \\
& \tilde{g}_{7}^{*}=3+\frac{174}{N}+\frac{7080}{N^{2}}+\ldots, \\
& \tilde{g}_{8}^{*}=84+\frac{6732}{N}+\frac{309204}{N^{2}}+\ldots
\end{aligned}
$$

\footnotetext{
${ }^{3}$ At finite $N$, using the beta functions given in the Appendix, we are able to find and study additional fixed points numerically. The analysis of behavior of the beta functions shows that they are all saddle points and, therefore, neither stable in the IR nor in the UV.
}

For the scaling dimension of $\phi$, we find from (A10):

$$
\begin{aligned}
\Delta_{\phi}= & \frac{d-2}{2}+\gamma_{\phi}=\frac{1}{2}-\frac{\epsilon}{2}+\epsilon^{2}\left(1-\frac{12}{N}+\frac{75}{N^{2}}+\ldots\right) \\
& +\mathcal{O}\left(\epsilon^{3}\right) .
\end{aligned}
$$

In the large $N$ limit, (5.8) is in agreement with the solution of the Schwinger-Dyson equation (4.1). For the scaling dimension of $\phi^{a b c} \phi^{a b c}$, we find

$$
\begin{aligned}
\Delta_{\phi^{2}}= & d-2+\gamma_{\phi^{2}}=1-\epsilon+32 \epsilon^{2}\left(1-\frac{12}{N}+\frac{75}{N^{2}}+\ldots\right) \\
& +\mathcal{O}\left(\epsilon^{3}\right) .
\end{aligned}
$$

In the large $N$ limit this is in agreement with (4.3). In general, calculating the $1 / N$ corrections in tensor models seems to be quite difficult [40], but it is nice to see that in the prismatic QFT the $3-\epsilon$ expansion provides us with explicit results for the $1 / N$ corrections to scaling dimensions of various operators.

The scaling dimension of the marginal prism operator is

$$
\begin{aligned}
\Delta_{\text {prism }}= & d+\frac{d \tilde{\beta}_{1}}{d \tilde{g}_{1}}=3-\epsilon-2 \epsilon+4 \epsilon \tilde{g}_{1}^{*}+\ldots=3+\epsilon \\
& +\mathcal{O}\left(\epsilon^{2}\right),
\end{aligned}
$$

which is in agreement with (4.4).

We have also performed two-loop calculations of the scaling dimensions of the tetrahedron and pillow operators; see the Appendix for the anomalous dimension matrix. In the large $N$ limit, we find

$$
\begin{aligned}
\Delta_{\text {tetra }} & =2(d-2)+\gamma_{\text {tetra }}=2+6 \epsilon+\mathcal{O}\left(\epsilon^{2}\right), \\
\Delta_{\text {pillow }} & =2(d-2)+\gamma_{\text {pillow }}=2-2 \epsilon+\mathcal{O}\left(\epsilon^{2}\right),
\end{aligned}
$$

which is in agreement with the Schwinger-Dyson result (4.8). Thus, we see that the large $N 3-\epsilon$ expansions from the Schwinger-Dyson approach have passed a number of 2-loop consistency checks.

We have also solved the equations for the fixed points of two-loop beta functions numerically for finite $N$. The results for the prismatic fixed point are shown in Table I. These results are in good agreement with the analytic $1 / N$ expansions (5.7) for $N \geq 200$. At $N=N_{\text {crit }}$, where $N_{\text {crit }} \approx 53.65$, the prismatic fixed point in $3-\epsilon$ dimensions merges with another fixed point ${ }^{4}$; they are located at

\footnotetext{
${ }^{4}$ This is similar, for example, to the situation in the $O(N)$ invariant cubic theory in $6-\epsilon$ dimensions [41,42], where $N_{\text {crit }} \approx 1038.266$. For general discussions of mergers of fixed points, see $[28,30]$.
} 
TABLE I. The numerical solutions for the coupling constants defined in (5.3).

\begin{tabular}{lccccccccc}
\hline \hline$N$ & $\tilde{g}_{1}^{*}$ & $\tilde{g}_{2}^{*}$ & $\tilde{g}_{3}^{*}$ & $\tilde{g}_{4}^{*}$ & $\tilde{g}_{5}^{*}$ & $\tilde{g}_{6}^{*}$ & $\tilde{g}_{7}^{*}$ & $\tilde{g}_{8}^{*}$ & $\gamma_{\phi} / \epsilon^{2}$ \\
\hline 54 & 0.89 & -33.06 & 7.87 & 83.69 & -11.13 & 6.86 & 27.37 & 2047.16 & 0.80 \\
100 & 0.94 & -37.56 & 6.23 & 55.35 & -11.53 & 6.28 & 5.98 & 212.08 & 0.89 \\
200 & 0.97 & -39.90 & 6.05 & 53.8 & -11.80 & 6.15 & 4.09 & 127.90 & 0.94 \\
400 & 0.99 & -40.99 & 6.01 & 53.78 & -11.91 & 6.08 & 3.48 & 103.03 & 0.97 \\
2000 & 1.00 & -41.81 & 6.00 & 53.94 & -11.98 & 6.02 & 3.09 & 87.45 & 0.99 \\
5000 & 1.00 & -41.92 & 6.00 & 53.97 & -11.99 & 6.01 & 3.04 & 85.36 & 0.998 \\
10000 & 1.00 & -41.96 & 6.00 & 53.99 & -12.00 & 6.00 & 3.02 & 84.68 & 0.999 \\
100000 & 1.00 & -42.00 & 6.00 & 54.00 & -12.00 & 6.00 & 3.00 & 84.07 & 1.00 \\
\hline \hline
\end{tabular}

$\tilde{g}_{1}^{*}=0.89, \quad \tilde{g}_{2}^{*}=-32.90, \quad \tilde{g}_{3}^{*}=8.24$,

$\tilde{g}_{4}^{*}=92.01, \quad \tilde{g}_{5}^{*}=-11.15, \quad \tilde{g}_{6}^{*}=7.00$,

$\tilde{g}_{7}^{*}=35.33, \quad \tilde{g}_{8}^{*}=3155.29$.

For $N<N_{\text {crit }}$ both of them become complex. For example, for $N=53.6$ the two complex fixed points are at

$\tilde{g}_{1}^{*}=0.89-0.0002 i, \quad \tilde{g}_{2}^{*}=-32.89+0.04 i$,

$\tilde{g}_{3}^{*}=8.24+0.15 i, \quad \tilde{g}_{4}^{*}=91.98+3.51 i$,

$\tilde{g}_{5}^{*}=-11.15-0.01 i, \quad \tilde{g}_{6}^{*}=7.00+0.06 i$,

$\tilde{g}_{7}^{*}=35.19+3.61 i, \quad \tilde{g}_{8}^{*}=3107.77+554.01 i$,

and at the complex conjugate values.

\section{BOSONIC QUANTUM MECHANICS}

The action (1.2) for $d=1$ describes the quantum mechanics of a particle moving in $N^{3}$ dimensions with a non-negative sextic potential which vanishes at the origin. ${ }^{5}$ Such a problem should exhibit a discrete spectrum with positive energy levels, and it is conceivable that in the large $N$ limit the gaps become exponentially small, leading to a nearly conformal behavior. For moderate values of $N$, this quantum mechanics problem may even be accessible to numerical studies.

Solving for the scaling dimensions of type A/C bilinears in $d=1$, we find that the low-lying eigenvalues are

$\Delta=1,1.57,2,3.29,4.12,5.36,6.14,7.38,8.15,9.39$,

$$
10.15,11.40, \ldots
$$

The plot for the eigenvalues is shown in Fig. 13.

\footnotetext{
${ }^{5}$ A very similar $d=1$ model with a stable sextic potential was studied in $[43,44]$ using the formulation [45] where a rank-3 tensor is viewed as $D$ matrices. It was argued $[43,44]$ that the sextic bosonic model does not have a good IR limit. We, however, do not find an obvious problem with the prismatic $d=1$ model because the complex scaling dimensions are absent for the bilinear operators. We note that the negative scaling dimension (2.15), which we find for $\phi$, is quite far from the $1 / 6$ mentioned in $[43,44]$.
}

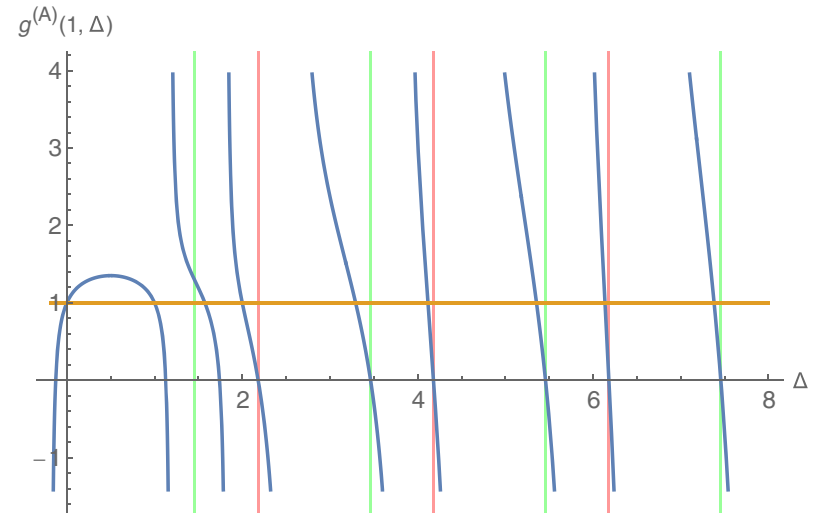

FIG. 13. The spectrum of scalar type A/C bilinears in $1 \mathrm{~d}$. Red vertical lines are asymptotes corresponding to $-2 \Delta_{\phi}+2 n$, and green vertical lines are asymptotes corresponding to $-2 \Delta_{\chi}+2 n$.

The smallest positive eigenvalue, $\Delta=1$, is the continuation of the solution $\Delta=d$ present for any $d$. As discussed in Sec. IV, it may correspond to a redundant operator. The next scaling dimension, $\Delta=1.57317$, may correspond to a mixture involving $\phi^{a b c} \phi^{a b c}$. The appearance of scaling dimension 2, which was also seen for the fermionic SYK and tensor models, means that the its dual ${ }^{6}$ should involve dilaton gravity in $\mathrm{AdS}_{2}$ [47-50].

Let us also list the type B scaling dimensions, i.e., the ones corresponding to operators $\phi^{a b c} \partial_{t}^{2 n} \chi^{a b c}$. Here we find real solutions $\Delta=1.01,2.96,4.94,6.93, \ldots$.

For large excitation numbers $n$, the type $\mathrm{A} / \mathrm{C}$ scaling dimensions appear to (slowly) approach $-2 \Delta_{\phi}+2 n$ and $-2 \Delta_{\chi}+2 n$ rather than $2 \Delta_{\phi}+2 n$ and $2 \Delta_{\chi}+2 n$, as shown in Fig. 4. The type $\mathrm{B}$ scaling dimensions also appear to slowly approach $-\Delta_{\phi}-\Delta_{\chi}+2 n$ rather than $\Delta_{\phi}+\Delta_{\chi}+2 n$. This is likely due to the fact that $\Delta_{\phi}$ is negative. Further work is needed to understand better the new features of the large $N$ solution in the regime where $d<1.35$ and $\Delta_{\phi}<0$.

\footnotetext{
${ }^{6}$ Of course, as observed in $[31,46]$, there are important differences between the holographic duals of tensor models and SYK models.
} 


\section{DISCUSSION}

In this paper we presented exact results for the $O(N)^{3}$ invariant theory (1.2) in the prismatic large $N$ limit where $g_{1} N^{3}$ is held fixed. This approach may be generalized to an $O(N)^{p}$ invariant theory of a rank- $p$ bosonic tensor $\phi^{a_{1} \ldots a_{p}}$, with odd $p \geq 3$. It has a positive potential of order $2 p$ :

$S_{2 p}=\int d^{d} x\left(\frac{1}{2}\left(\partial_{\mu} \phi^{a b c}\right)^{2}+\frac{g_{1}}{(2 p) !}\left(\phi^{p}\right)^{a_{1} \ldots a_{p}}\left(\phi^{p}\right)^{a_{1} \ldots a_{p}}\right)$.

To solve these models in the large $N$ limit where $g_{1} N^{p}$ is held fixed, we may rewrite the action with the help of an additional tensor field $\chi$ :

$$
\begin{aligned}
S= & \int d^{d} x\left(\frac{1}{2}\left(\partial_{\mu} \phi^{a b c}\right)^{2}+\frac{g}{p !}\left(\phi^{p}\right)^{a_{1} \ldots a_{p}} \chi^{a_{1} \ldots a_{p}}\right. \\
& \left.-\frac{1}{2} \chi^{a_{1} \ldots a_{p}} \chi^{a_{1} \ldots a_{p}}\right) .
\end{aligned}
$$

For discussions of the structure of the interaction vertex with odd $p>3$, see $[5,51,52]$. The models (7.1) are tensor counterparts of the SYK-like models introduced in [16]; therefore, the Schwinger-Dyson equations derived there should be applicable to the tensor models. It would be interesting to study the large $N$ solution of theories with $p>3$ in more detail using methods analogous to the ones used for $p=3$.

In this paper we analyzed the renormalization of the prismatic theory at the two-loop order, using the beta functions in [36,37]. The general four-loop terms are also given there, and it would be interesting to study the effects they produce. It should be possible to extend the calculations to even higher loops by modifying the calculations in [39] to an arbitrary tensorial interaction, which we leave as a possible avenue for future work. In this context, it would also be interesting to study the possibility of fixed points with other large $N$ scalings, perhaps dominated by the "wheel" interaction $\left(g_{2}\right)$ of Fig. 1, in addition to the large $N$ fixed point dominated by the prism interaction $\left(g_{1}\right)$ studied in this paper. ${ }^{7}$

Another interesting extension of the $O(N)^{3}$ symmetric model (1.2) is to add a 2-component Majorana fermion $\psi^{a b c}$, so that the fields can be assembled into a $d=3$ $\mathcal{N}=1$ superfield

$$
\Phi^{a b c}=\phi^{a b c}+\bar{\theta} \psi^{a b c}+\bar{\theta} \theta \chi^{a b c} .
$$

Then the prismatic scalar potential follows if we assume a tetrahedral superpotential for $\Phi^{a b c}$ [5]. Large $N$ treatments

\footnotetext{
${ }^{7} \mathrm{~A} d=0$ theory with wheel interactions was studied in [53].
}

of supersymmetric tensor and SYK-like models with two supercharges have been given in [16,25], and we expect the solution of the $\mathcal{N}=1$ supertensor model in $d<3$ to work analogously. An advantage of the tensor QFT approach is that one can also develop the $3-\epsilon$ expansion using the standard renormalized perturbation theory. In the supersymmetric case, it is sufficient to introduce only three coupling constants:

$$
\begin{aligned}
W= & g_{1} \Phi^{a_{1} b_{1} c_{1}} \Phi^{a_{1} b_{2} c_{2}} \Phi^{a_{2} b_{1} c_{2}} \Phi^{a_{2} b_{2} c_{1}} \\
& +g_{2}\left(\Phi^{a_{1} b_{1} c_{1}} \Phi^{a_{1} b_{1} c_{2}} \Phi^{a_{2} b_{2} c_{1}} \Phi^{a_{2} b_{2} c_{2}}\right. \\
& +\Phi^{a_{1} b_{1} c_{1}} \Phi^{a_{2} b_{1} c_{1}} \Phi^{a_{1} b_{2} c_{2}} \Phi^{a_{2} b_{2} c_{2}} \\
& \left.+\Phi^{a_{1} b_{1} c_{1}} \Phi^{a_{1} b_{2} c_{1}} \Phi^{a_{2} b_{1} c_{2}} \Phi^{a_{2} b_{2} c_{2}}\right) \\
& +g_{3} \Phi^{a_{1} b_{1} c_{1}} \Phi^{a_{1} b_{1} c_{1}} \Phi^{a_{2} b_{2} c_{2}} \Phi^{a_{2} b_{2} c_{2}}
\end{aligned}
$$

and it is possible to find explicit expressions for the beta functions and operator scaling dimensions [54]. Also, directly in $d=3$ it is possible to couple the $\mathcal{N}=1$ theory with the above superpotential to $O(N)_{k_{1}} \times O(N)_{k_{2}} \times$ $O(N)_{k_{3}}$ supersymmetric Chern-Simons gauge theory with levels $k_{1}, k_{2}, k_{3}$, and derive the corresponding beta functions for couplings $g_{i}$ [54].

\section{ACKNOWLEDGMENTS}

We are grateful to C.-M. Chang, M. Rangamani, D. Stanford, E. Witten and J. Yoon for useful discussions. I. R. K. thanks the Yukawa Institute for Theoretical Physics where some of his work on this paper was carried out during the workshop YITP-T-18-04 "New Frontiers in String Theory 2018". S. P. thanks the Princeton Center for Theoretical Science for hospitality as well as the International Centre for Theoretical Sciences, Bengaluru where some of his work on this paper was carried out during the program "AdS/CFT at 20 and Beyond" (ICTS/ adscft20/2018/05). The work of S. G. was supported in part by the US NSF under Grant No. PHY-1620542. The work of I. R. K. and F. P. was supported in part by the US NSF under Grant No. PHY-1620059. The work of S.P. was supported in part by a DST-SERB Early Career Research Award (ECR/2017/001023) and a DST INSPIRE Faculty Award. The work of G. T. was supported in part by the MURI Grant No. W911NF-14-1-0003 from ARO and by DOE Grant No. de-sc0007870.

\section{APPENDIX: THE TWO-LOOP BETA FUNCTIONS AND ANOMALOUS DIMENSIONS}

In this Appendix we state our explicit two-loop results for the $O(N)^{3}$ invariant theory with the 8 coupling constants and interaction terms 


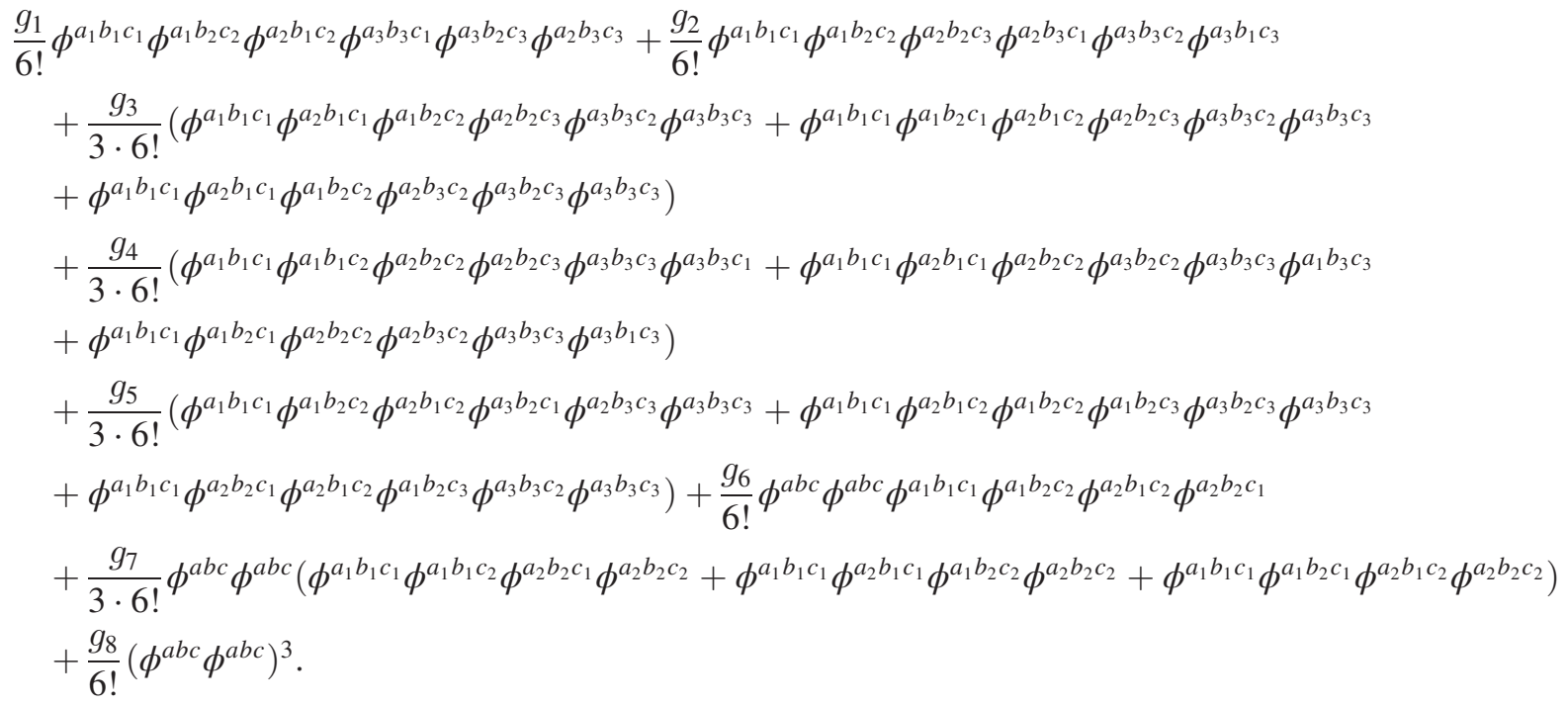

We find

$$
\begin{aligned}
\beta_{1}= & -2 g_{1} \epsilon+\frac{1}{270(8 \pi)^{2}}\left(\left(g_{5}^{2}+3\left(g_{1}^{2}+8 g_{6}^{2}\right)\right) N^{3}+3\left(3 g_{5}^{2}+4\left(2 g_{1}+3 g_{2}+4 g_{6}\right) g_{5}+6 g_{1}\left(g_{1}+3 g_{2}\right)\right) N^{2}\right. \\
& +2\left(32 g_{5}^{2}+\left(90 g_{1}+72 g_{2}+96 g_{6}\right) g_{5}+6 g_{4}\left(9 g_{2}+4 g_{5}\right)+9\left(5 g_{1}^{2}+6 g_{2} g_{1}+16 g_{6} g_{1}+8 g_{7} g_{1}+9 g_{2}^{2}\right.\right. \\
& \left.\left.+24 g_{2} g_{6}\right)\right) N+2 g_{3}^{2}(N(N+6)+55)+2 g_{3}\left(9 N\left(g_{1}(N+8)+8 g_{2}\right)+6 g_{4}(N+6)\right. \\
& \left.+2 g_{5}(N+10)(2 N+5)+2\left(60 g_{1}+63 g_{2}+96 g_{6}+16 g_{7}\right)\right)+2\left(36 g_{4}^{2}+36\left(5 g_{1}+3 g_{2}+2 g_{5}\right) g_{4}\right. \\
& +80 g_{5}^{2}+4 g_{5}\left(45 g_{1}+4\left(9 g_{2}+6 g_{6}+8 g_{7}\right)\right)+3\left(34 g_{1}^{2}+12\left(7 g_{2}+4 g_{6}+2 g_{7}+20 g_{8}\right) g_{1}+27 g_{2}^{2}\right. \\
& \left.\left.+128 g_{6}^{2}+48 g_{2}\left(g_{6}+2 g_{7}\right)\right)\right),
\end{aligned}
$$

$$
\begin{aligned}
\beta_{2}= & -2 g_{2} \epsilon+\frac{1}{270(8 \pi)^{2}}\left(g_{5}\left(12 g_{1}+g_{5}\right) N^{2}+2\left(13 g_{5}^{2}+18\left(g_{1}+g_{2}\right) g_{5}+9 g_{1}\left(g_{1}+2 g_{4}+8 g_{6}\right)+72 g_{2} g_{7}\right) N\right. \\
& +2 g_{3}^{2}(N(N+6)+19)+2 g_{3}\left(3 N\left(3 g_{2}(N+4)+8 g_{1}\right)+6 g_{4}(N+2)+6 g_{5}(N+6)+30 g_{1}+36 g_{2}\right. \\
& \left.+32 g_{7}\right)+2\left(36 g_{1}^{2}+54 g_{2} g_{1}+96 g_{7} g_{1}+45 g_{2}^{2}+12 g_{4}^{2}+20 g_{5}^{2}+12 g_{4}\left(3 g_{1}+9 g_{2}+2 g_{5}\right)\right. \\
& \left.\left.+12 g_{5}\left(4 g_{1}+3 g_{2}+8 g_{6}\right)+72 g_{2} g_{7}+720 g_{2} g_{8}\right)\right),
\end{aligned}
$$

$$
\begin{aligned}
\beta_{3}= & -2 g_{3} \epsilon+\frac{1}{270(8 \pi)^{2}}\left(2\left(g_{5}^{2}+8 g_{7}^{2}\right) N^{3}+3\left(6 g_{1}^{2}+12 g_{5} g_{1}+27 g_{2}^{2}+5 g_{5}^{2}\right) N^{2}+2\left(83 g_{5}^{2}+2\left(66 g_{1}\right.\right.\right. \\
& \left.\left.+63 g_{2}+48 g_{6}+64 g_{7}\right) g_{5}+9\left(2 g_{1}+3 g_{2}\right)\left(4 g_{1}+3 g_{2}\right)+96\left(g_{1}+3 g_{2}\right) g_{7}\right) N+g_{3}^{2}(N(N(2 N+31) \\
& +244)+388)+18 g_{4}^{2}(N(N+16)+12)+12 g_{4}\left(3 g_{1}(N+1)(N+14)+g_{5}(5 N(N+6)+72)\right. \\
& \left.+(N+2)\left(9 g_{2}(N+3)+8 g_{7} N\right)+96 g_{6}+64 g_{7}\right)+4 g_{3}\left(3 g_{4}(N(N(N+6)+28)+102)\right. \\
& +N\left(g_{5}(11 N+74)+6\left(g_{1}+3 g_{2}+4 g_{7}\right) N+66 g_{1}+72 g_{2}+60 g_{6}+84 g_{7}\right)+194 g_{5} \\
& \left.+3\left(71 g_{1}+81 g_{2}+32 g_{6}+76 g_{7}+120 g_{8}\right)\right)+4\left(92 g_{5}^{2}+2\left(93 g_{1}+90 g_{2}+72 g_{6}+80 g_{7}\right) g_{5}\right. \\
& \left.\left.+128 g_{7}^{2}+9\left(7 g_{1}^{2}+15 g_{2} g_{1}+9 g_{2}^{2}+24\left(g_{1}+g_{2}\right) g_{6}\right)+144\left(g_{1}+g_{2}\right) g_{7}\right)\right),
\end{aligned}
$$




$$
\begin{aligned}
\beta_{4}= & -2 g_{4} \epsilon+\frac{1}{270(8 \pi)^{2}}\left(\left(g_{5}^{2}+8 g_{7}^{2}\right) N^{3}+4 g_{5}\left(3 g_{1}+g_{5}\right) N^{2}+6\left(3 g_{1}^{2}+9 g_{5}^{2}+8\left(g_{1}+3 g_{2}\right) g_{7}\right.\right. \\
& \left.+2 g_{5}\left(5 g_{1}+9 g_{2}+4\left(g_{6}+3 g_{7}\right)\right)\right) N+2 g_{3}^{2}(N(N(N+7)+34)+113)+9 g_{4}^{2}\left(N(N+2)^{2}+52\right) \\
& +4 g_{3}\left(9 g_{2}(N+2)^{2}+3 g_{1}(N+1)(N+13)+N\left(g_{4}(6 N+75)+g_{5}(6 N+31)+8 g_{7}(N+4)\right)\right. \\
& \left.+16\left(3 g_{4}+5 g_{5}+6 g_{6}+5 g_{7}\right)\right)+12 g_{4}\left(3 g_{1}(N+12)+2 g_{5}(N(N+6)+13)+8 N\left(g_{7}(N+2)+3 g_{6}\right)\right. \\
& \left.+48 g_{2}+44 g_{7}+120 g_{8}\right)+2\left(54 g_{1}^{2}+162 g_{2} g_{1}+96 g_{7} g_{1}+81 g_{2}^{2}+58 g_{5}^{2}+128 g_{7}^{2}\right. \\
& \left.\left.+4 g_{5}\left(27 g_{1}+27 g_{2}+24 g_{6}+32 g_{7}\right)\right)\right),
\end{aligned}
$$

$$
\begin{aligned}
\beta_{5}= & -2 g_{5} \epsilon+\frac{2}{270(8 \pi)^{2}}\left(\left(3\left(g_{1} g_{5}+8 g_{6} g_{7}\right) N^{3}+2\left(9 g_{1}^{2}+9\left(3 g_{2}+g_{5}\right) g_{1}+g_{5}\left(27 g_{2}+6 g_{5}+16 g_{7}\right)\right) N^{2}\right.\right. \\
& +\left(99 g_{1}^{2}+6\left(45 g_{2}+35 g_{5}+36 g_{6}+40 g_{7}\right) g_{1}+81 g_{2}^{2}+216 g_{2}\left(g_{5}+g_{6}\right)\right. \\
& \left.+4 g_{5}\left(21 g_{5}+42 g_{6}+38 g_{7}\right)\right) N+g_{3}^{2}(N(5 N+52)+161)+36 g_{4}^{2}(N+3) \\
& +3 g_{4}\left(12 g_{1}(N(N+5)+12)+g_{5}(N(N(N+6)+52)+132)+6(N+2)\left(4 g_{6} N+9 g_{2}\right)\right. \\
& \left.+96 g_{6}+64 g_{7}\right)+2 g_{3}\left(6 g_{1}(N(3 N+16)+37)+9 g_{2}(10 N+23)+N\left(g_{4}(6 N+39)\right.\right. \\
& \left.\left.+g_{5}(N(N+13)+97)+24 g_{6}(N+4)\right)+6\left(23 g_{4}+33 g_{5}+32 g_{6}+24 g_{7}\right)\right)+270 g_{1}^{2} \\
& +243 g_{2}^{2}+212 g_{5}^{2}+432 g_{1} g_{2}+444 g_{1} g_{5}+504 g_{2} g_{5}+432 g_{1} g_{6}+432 g_{2} g_{6} \\
& \left.\left.+384 g_{5} g_{6}+384 g_{1} g_{7}+288 g_{2} g_{7}+328 g_{5} g_{7}+768 g_{6} g_{7}+720 g_{5} g_{8}\right)\right), \\
& \\
\beta_{6}= & -2 g_{6} \epsilon+\frac{2}{270(8 \pi)^{2}}\left(2\left(g_{5} g_{7}+3 g_{6}\left(g_{1}+12 g_{8}\right)\right) N^{3}+\left(6\left(9 g_{2} g_{6}+4\left(g_{1}+2 g_{6}\right) g_{7}\right)\right.\right. \\
& \left.+g_{5}\left(3 g_{1}+12 g_{6}+10 g_{7}+72 g_{8}\right)\right) N^{2}+\left(7 g_{5}^{2}+2\left(3 g_{1}+9 g_{2}+12 g_{6}+32 g_{7}+72 g_{8}\right) g_{5}\right. \\
& \left.+3\left(3 g_{1}+12\left(2 g_{6}+g_{7}+12 g_{8}\right) g_{1}+48 g_{6}^{2}+8\left(3 g_{2}+5 g_{6}\right) g_{7}\right)\right) N+g_{3}^{2}(4 N+6) \\
& +3 g_{4}\left(12 g_{1} N+g_{5}(N(N+6)+10)+4 g_{7}(N+2)+18 g_{2}+60 g_{6}\right)+2 g_{3}\left(6 g_{1}(N+4)\right. \\
& \left.+g_{5}(N(N+6)+19)+3 g_{6}(N(N+10)+4)+2 g_{7} N(N+5)+9 g_{2}+21 g_{4}+18\left(g_{7}+4 g_{8}\right)\right) \\
& +13 g_{5}^{2}+48 g_{7}^{2}+36 g_{1} g_{2}+30 g_{1} g_{5}+18 g_{2} g_{5}+48 g_{1} g_{6}+72 g_{2} g_{6}+108 g_{5} g_{6}+120 g_{1} g_{7} \\
& \left.+36 g_{2} g_{7}+92 g_{5} g_{7}+120 g_{6} g_{7}+432 g_{2} g_{8}+144 g_{5} g_{8}+1296 g_{6} g_{8}\right),
\end{aligned}
$$

$$
\begin{aligned}
\beta_{7}= & -2 g_{7} \epsilon+\frac{1}{270(8 \pi)^{2}}\left(4\left(3 g_{5} g_{6}+g_{7}\left(2 g_{3}+3 g_{4}+36 g_{8}\right)\right) N^{3}+\left(10 g_{3}^{2}+24\left(g_{4}+3 g_{6}\right.\right.\right. \\
& \left.+2\left(g_{7}+6 g_{8}\right)\right) g_{3}+7 g_{5}^{2}+3\left(9 g_{4}^{2}+8\left(3 g_{6}+2\left(g_{7}+9 g_{8}\right)\right) g_{4}+8\left(5 g_{7}^{2}+\left(g_{1}+3 g_{2}+2 g_{5}\right) g_{7}\right.\right. \\
& \left.\left.\left.+\left(3 g_{1}+g_{5}\right) g_{6}\right)\right)\right) N^{2}+\left(9 g_{1}^{2}+54 g_{5} g_{1}+72 g_{6} g_{1}+216 g_{7} g_{1}+48 g_{3}^{2}+63 g_{4}^{2}+22 g_{5}^{2}+216 g_{6}^{2}\right. \\
& +216 g_{7}^{2}+216 g_{2} g_{6}+216 g_{5} g_{6}+144 g_{2} g_{7}+160 g_{5} g_{7}+576 g_{6} g_{7}+144\left(3 g_{1}+9 g_{2}+5 g_{5}\right) g_{8} \\
& +6 g_{4}\left(6 g_{1}+18 g_{2}+21 g_{5}+36 g_{6}+52 g_{7}+72 g_{8}\right)+4 g_{3}\left(3 g_{1}+9 g_{2}+36 g_{4}+19 g_{5}+42 g_{6}\right. \\
& \left.\left.+90 g_{7}+144 g_{8}\right)\right) N+2\left(27 g_{1}^{2}+3\left(9 g_{2}+23 g_{3}+30 g_{4}+12 g_{5}+48 g_{6}+40 g_{7}+144 g_{8}\right) g_{1}\right. \\
& +9 g_{2}\left(7 g_{3}+6\left(g_{5}+2 g_{6}+4 g_{7}\right)\right)+2\left(31 g_{3}^{2}+\left(81 g_{4}+50 g_{5}+114 g_{6}+112 g_{7}+216 g_{8}\right) g_{3}\right. \\
& +54 g_{4}^{2}+21 g_{5}^{2}+108 g_{6}^{2}+96 g_{7}^{2}+66 g_{5} g_{6}+106 g_{5} g_{7}+144 g_{6} g_{7}+72\left(2 g_{5}+9 g_{7}\right) g_{8} \\
& \left.\left.\left.+3 g_{4}\left(17 g_{5}+36 g_{6}+66 g_{7}+72 g_{8}\right)\right)\right)\right),
\end{aligned}
$$




$$
\begin{aligned}
\beta_{8}= & -2 g_{8} \epsilon+\frac{1}{270(8 \pi)^{2}}\left(2 \left(g_{5}\left(2\left(3 g_{6}\left(N^{2}+N+3\right)+7 g_{7}(N+1)+36 g_{8}\right)+3 g_{1}\right)+2\left(3 g_{6}^{2} N^{3}\right.\right.\right. \\
& +g_{7}^{2} N^{3}+18 g_{8}^{2}\left(3 N^{3}+22\right)+3 g_{7}^{2} N^{2}+12 g_{6} g_{7} N^{2}+72 g_{8}\left(g_{7}\left(N^{2}+N+1\right)+3 g_{6} N\right)+9 g_{6}^{2} N \\
& \left.+21 g_{7}^{2} N+12 g_{6} g_{7} N+g_{1}\left(9 g_{6} N+6 g_{7}\right)+6 g_{6}^{2}+23 g_{7}^{2}+9 g_{2} g_{6}+48 g_{6} g_{7}\right)+g_{5}^{2}(N+1) \\
& \left.+3 g_{4}\left(2\left(6 g_{6} N+g_{7}(N(N+3)+5)+36 g_{8}\right)+3 g_{5}\right)\right)+g_{3}^{2}(2 N+9)+4 g_{3}\left(3 g_{4} N+3 g_{6}(2 N+5)\right. \\
& \left.\left.+2 g_{7}(N(N+3)+7)+36 g_{8} N+2 g_{5}\right)+9 g_{2}^{2}+39 g_{4}^{2}\right),
\end{aligned}
$$

and

$$
\begin{aligned}
& \gamma_{\phi}=\frac{1}{12 \cdot 90^{2}(8 \pi)^{4}}\left(\left(3 g_{1}^{2}+9 g_{2}^{2}+g_{3}^{2}+3 g_{4}^{2}+g_{5}^{2}+12 g_{6}^{2}+4 g_{7}^{2}+72 g_{8}^{2}\right) N^{6}+\left(6 g_{3}^{2}+2\left(3 g_{1}+9 g_{2}\right.\right.\right. \\
& \left.+6\left(g_{4}+g_{5}\right)+8 g_{7}\right) g_{3}+9 g_{4}^{2}+5 g_{5}^{2}+12 g_{7}^{2}+54 g_{1} g_{2}+24 g_{1} g_{5}+24 g_{5} g_{6}+48 g_{6} g_{7} \\
& \left.+12 g_{4}\left(g_{5}+2 g_{7}\right)+144 g_{7} g_{8}\right) N^{5}+\left(81 g_{1}^{2}+12\left(9 g_{3}+6 g_{4}+5 g_{5}+12 g_{6}+2 g_{7}\right) g_{1}+81 g_{2}^{2}\right. \\
& +39 g_{3}^{2}+27 g_{4}^{2}+51 g_{5}^{2}+36 g_{6}^{2}+84 g_{7}^{2}+108 g_{3} g_{4}+76 g_{3} g_{5}+72 g_{4} g_{5}+96 g_{3} g_{6}+144 g_{4} g_{6} \\
& +48 g_{5} g_{6}+80 g_{3} g_{7}+96 g_{4} g_{7}+88 g_{5} g_{7}+48 g_{6} g_{7}+36 g_{2}\left(2 g_{3}+g_{4}+4 g_{5}+2 g_{7}\right) \\
& \left.+144\left(g_{3}+g_{4}+3 g_{6}+g_{7}\right) g_{8}\right) N^{4}+\left(102 g_{1}^{2}+6\left(75 g_{2}+47 g_{3}+54 g_{4}+64 g_{5}+24 g_{6}\right.\right. \\
& \left.+68 g_{7}+24 g_{8}\right) g_{1}+54 g_{2}^{2}+160 g_{3}^{2}+171 g_{4}^{2}+143 g_{5}^{2}+120 g_{6}^{2}+148 g_{7}^{2}+432 g_{8}^{2}+288 g_{3} g_{4} \\
& +344 g_{3} g_{5}+336 g_{4} g_{5}+336 g_{3} g_{6}+288 g_{4} g_{6}+360 g_{5} g_{6}+336 g_{3} g_{7}+336 g_{4} g_{7}+296 g_{5} g_{7} \\
& \left.+336 g_{6} g_{7}+144\left(2 g_{3}+3\left(g_{4}+g_{5}\right)+g_{7}\right) g_{8}+18 g_{2}\left(19 g_{3}+24 g_{4}+14 g_{5}+32 g_{6}+12 g_{7}+24 g_{8}\right)\right) N^{3} \\
& +2\left(189 g_{1}^{2}+6\left(45 g_{2}+58 g_{3}+66 g_{4}+49 g_{5}+72 g_{6}+54 g_{7}+108 g_{8}\right) g_{1}+216 g_{2}^{2}+177 g_{3}^{2}\right. \\
& +189 g_{4}^{2}+176 g_{5}^{2}+216 g_{6}^{2}+120 g_{7}^{2}+318 g_{3} g_{4}+330 g_{3} g_{5}+336 g_{4} g_{5}+360 g_{3} g_{6}+288 g_{4} g_{6} \\
& +312 g_{5} g_{6}+328 g_{3} g_{7}+312 g_{4} g_{7}+372 g_{5} g_{7}+336 g_{6} g_{7}+72\left(4 g_{3}+4 g_{4}+5 g_{5}+4 g_{7}\right) g_{8} \\
& \left.+18 g_{2}\left(17 g_{3}+19 g_{4}+20 g_{5}+12 g_{6}+26 g_{7}+12 g_{8}\right)\right) N^{2}+4\left(81 g_{1}^{2}+3\left(63 g_{2}+63 g_{3}\right.\right. \\
& \left.+51 g_{4}+64 g_{5}+60 g_{6}+70 g_{7}+36 g_{8}\right) g_{1}+81 g_{2}^{2}+87 g_{3}^{2}+72 g_{4}^{2}+90 g_{5}^{2}+72 g_{6}^{2}+96 g_{7}^{2} \\
& +207 g_{3} g_{4}+185 g_{3} g_{5}+189 g_{4} g_{5}+156 g_{3} g_{6}+216 g_{4} g_{6}+204 g_{5} g_{6}+184 g_{3} g_{7}+174 g_{4} g_{7} \\
& +182 g_{5} g_{7}+168 g_{6} g_{7}+36\left(6 g_{3}+3 g_{4}+5 g_{5}+12 g_{6}+4 g_{7}\right) g_{8}+9 g_{2}\left(23 g_{3}+18 g_{4}+19 g_{5}\right. \\
& \left.\left.+24 g_{6}+18 g_{7}+36 g_{8}\right)\right) N+4\left(48 g_{1}^{2}+\left(90 g_{2}+78 g_{3}+90 g_{4}+84 g_{5}+72 g_{6}+60 g_{7}+72 g_{8}\right) g_{1}\right. \\
& +45 g_{2}^{2}+43 g_{3}^{2}+51 g_{4}^{2}+42 g_{5}^{2}+48 g_{6}^{2}+52 g_{7}^{2}+144 g_{8}^{2}+72 g_{3} g_{4}+82 g_{3} g_{5}+78 g_{4} g_{5}+96 g_{3} g_{6} \\
& +72 g_{4} g_{6}+72 g_{5} g_{6}+84 g_{3} g_{7}+96 g_{4} g_{7}+76 g_{5} g_{7}+96 g_{6} g_{7}+18 g_{2}\left(4 g_{3}+5 g_{4}+5 g_{5}+4\left(g_{6}+g_{7}\right)\right) \\
& \left.\left.+72\left(g_{3}+2 g_{4}+g_{5}+2 g_{7}\right) g_{8}\right)\right) \text {. }
\end{aligned}
$$

At the two-loop level we also find the relation $\gamma_{\phi^{2}}=32 \gamma_{\phi}$.

We can study the anomalous dimensions for quartic operators

$$
\begin{aligned}
& O_{1}=O_{\text {tetra }}=\phi^{a_{1} b_{1} c_{1}} \phi^{a_{1} b_{2} c_{2}} \phi^{a_{2} b_{1} c_{2}} \phi^{a_{2} b_{2} c_{1}}, \\
& O_{2}=O_{\text {pillow }}=\frac{1}{3}\left(\phi_{a_{1} b_{1} c_{1}} \phi_{a_{2} b_{1} c_{1}} \phi_{a_{1} b_{2} c_{2}} \phi_{a_{2} b_{2} c_{2}}+\phi_{a_{1} b_{1} c_{1}} \phi_{a_{1} b_{2} c_{1}} \phi_{a_{2} b_{1} c_{2}} \phi_{a_{2} b_{2} c_{2}}+\phi_{a_{1} b_{1} c_{1}} \phi_{a_{1} b_{1} c_{2}} \phi_{a_{2} b_{2} c_{1}} \phi_{a_{2} b_{2} c_{2}}\right) \\
& O_{3}=O_{\text {d.t. }}=\phi^{a_{1} b_{1} c_{1}} \phi^{a_{1} b_{1} c_{1}} \phi^{a_{2} b_{2} c_{2}} \phi^{a_{2} b_{2} c_{2}} .
\end{aligned}
$$


The matrix of anomalous dimensions for quartic operators can be written in the following way:

$$
\begin{aligned}
\gamma_{O}^{11}= & \frac{1}{720 \pi^{2}}\left(2\left(6 g_{1}+2 g_{3}+3 g_{4}+5 g_{5}+2 g_{7}+12 g_{8}\right)+g_{1}\left(N^{3}+12 N+8\right)+4\left(g_{5}+3 g_{6}+g_{7}\right) N\right. \\
& \left.+9 g_{2} N^{2}+2 g_{5} N^{2}+g_{3}\left(6 N+N^{2}\right)\right) \\
\gamma_{O}^{12}= & \frac{1}{2160 \pi^{2}}\left(2\left(9 g_{2}+9 g_{3}+6 g_{4}+11 g_{5}+12 g_{6}+8 g_{7}\right)+6 g_{1}\left(6+3 N+2 N^{2}\right)+36 g_{2} N+6 g_{4} N\right. \\
& \left.+12 g_{6}\left(2 N+N^{2}\right)+2 g_{3}\left(5 N+N^{2}\right)+g_{5}\left(24 N+5 N^{2}+N^{3}\right)\right) \\
\gamma_{O}^{13}= & \frac{1}{180 \pi^{2}}\left(6 g_{2}+2 g_{3}+6 g_{1} N+g_{6}\left(8+N^{3}\right)+g_{5}\left(2+2 N+N^{2}\right)\right) \\
\gamma_{O}^{21}= & \frac{1}{720 \pi^{2}}\left(2\left(12 g_{1}+9 g_{2}+11 g_{3}+12 g_{4}+9 g_{5}+12 g_{6}+8 g_{7}\right)+g_{5} N^{3}\right. \\
& \left.+2\left(3 g_{1}+9 g_{2}+7 g_{3}+9 g_{4}+9 g_{5}+6 g_{6}+10 g_{7}\right) N+2\left(3\left(g_{1}+g_{3}+g_{4}\right)+g_{5}\right) N^{2}\right) \\
\gamma_{O}^{22}= & \frac{1}{2160 \pi^{2}}\left(64 g_{3}+66 g_{4}+62 g_{5}+48 g_{6}+60 g_{7}+72 g_{8}+6 g_{1}(N+1)(N+8)+18 g_{2}\left(4+2 N+N^{2}\right)\right. \\
& \left.+3 g_{4}\left(18 N+4 N^{2}+N^{3}\right)+2 g_{3}\left(27 N+6 N^{2}+N^{3}\right)+4\left(6 g_{6} N+4 g_{7}\left(2 N+N^{2}\right)+g_{5}\left(10 N+3 N^{2}\right)\right)\right) \\
\gamma_{O}^{23}= & \frac{1}{180 \pi^{2}}\left(6 g_{3}+6 g_{4}+4 g_{5}+8 g_{7}+3 g_{1}(N+2)+9 g_{2} N+5 g_{5} N+g_{7} N^{3}+3 g_{4}\left(N^{2}+N\right)+2 g_{3}\left(2 N+N^{2}\right)\right) \\
\gamma_{O}^{31}= & \frac{1}{720 \pi^{2}}\left(3 g_{2}+3 g_{5}+4 g_{6}+8 g_{7}+3 g_{1} N+g_{3}(5+2 N)+6 g_{4} N+g_{5}\left(N^{2}+N\right)+4\left(g_{7} N+9 g_{8} N+g_{7} N^{2}\right)\right. \\
& \left.+2 g_{6}\left(3 N+N^{3}\right)\right) \\
\gamma_{O}^{32}= & \frac{1}{2160 \pi^{2}}\left(6 g_{1}+7 g_{5}+24 g_{6}+22 g_{7}+36 g_{8}+2 g_{3}\left(5+3 N+N^{2}\right)+3 g_{4}\left(5+3 N+N^{2}\right)+7 g_{5} N\right. \\
& \left.+12 g_{6}\left(N+N^{2}\right)+36 g_{8}\left(N+N^{2}\right)+2 g_{7}\left(13 N+3 N^{2}+N^{3}\right)\right) .
\end{aligned}
$$

The results for the quartic operator dimensions in the prismatic large $N$ limit are listed in (5.11).

A consistent truncation of the system of eight coupling constants is to keep only $g_{8}$ nonvanishing, since the tripletrace term is the only one which has $O\left(N^{3}\right)$ symmetry. Then we find

$$
\begin{aligned}
& \beta_{8}=-2 g_{8} \epsilon+\frac{1}{15(8 \pi)^{2}} g_{8}^{2}\left(3 N^{3}+22\right), \\
& \gamma_{\phi}=\frac{1}{1350(8 \pi)^{4}} g_{8}^{2}\left(N^{3}+2\right)\left(N^{3}+4\right),
\end{aligned}
$$

in agreement with [37,39]. Thus, there is a fixed point with

$$
\begin{aligned}
g_{8}^{*} & =\frac{30(8 \pi)^{2} \epsilon}{3 N^{3}+22}, \\
g_{i}^{*} & =0, \\
i & =1, \ldots, 7 .
\end{aligned}
$$

At this fixed point,

$$
\partial \beta_{8} / \partial g_{8}=-2 \epsilon+\frac{2}{15(8 \pi)^{2}} g_{8}^{*}\left(3 N^{3}+22\right)=2 \epsilon+\mathcal{O}\left(\epsilon^{2}\right),
$$

so the triple-trace operator is irrelevant. However, the other 7 operators appear to be relevant for sufficiently large $N$. For example,

$$
\frac{\partial \beta_{1}}{\partial g_{1}}=-2 \epsilon+\frac{2 g_{8}^{*}}{9(8 \pi)^{2}}=\epsilon\left(-2+\frac{20}{3\left(3 N^{3}+22\right)}\right)+\mathcal{O}\left(\epsilon^{2}\right) .
$$

So, this fixed point has 7 unstable directions. Examination of 4-loop and higher corrections [37,39] shows that the $3-\epsilon$ expansions of operator dimensions at this fixed point do not generally have a finite large $N$ limit starting with order $\epsilon^{3}$. This is in contrast with the prismatic fixed point where all the $g_{i}^{*}$ are nonvanishing and scale as (5.3); as a result, the large $N$ limit is smooth. 
[1] R. Gurau, Colored group field theory, Commun. Math. Phys. 304, 69 (2011).

[2] V. Bonzom, R. Gurau, A. Riello, and V. Rivasseau, Critical behavior of colored tensor models in the large N limit, Nucl. Phys. B853, 174 (2011).

[3] S. Carrozza and A. Tanasa, $O(N)$ Random tensor models, Lett. Math. Phys. 106, 1531 (2016).

[4] E. Witten, An SYK-Like Model without disorder, arXiv:1610.09758.

[5] I. R. Klebanov and G. Tarnopolsky, Uncolored random tensors, melon diagrams, and the Sachdev-Ye-Kitaev models, Phys. Rev. D 95, 046004 (2017).

[6] I. R. Klebanov, F. Popov, and G. Tarnopolsky, TASI lectures on large $N$ tensor models, Proc. Sci. TASI2017 (2018) 004.

[7] S. Sachdev and J. Ye, Gapless Spin Fluid Ground State in a Random, Quantum Heisenberg Magnet, Phys. Rev. Lett. 70, 3339 (1993).

[8] A. Kitaev, A simple model of quantum holography, http:// online.kitp.ucsb.edu/online/entangled15/kitaev/, http:// online.kitp.ucsb.edu/online/entangled15/kitaev2/, KITP, 2015.

[9] J. Polchinski and V. Rosenhaus, The spectrum in the Sachdev-Ye-Kitaev model, J. High Energy Phys. 04 (2016) 001.

[10] J. Maldacena and D. Stanford, Comments on the SachdevYe-Kitaev model, Phys. Rev. D 94, 106002 (2016).

[11] A. Jevicki, K. Suzuki, and J. Yoon, Bi-local holography in the SYK model, J. High Energy Phys. 07 (2016) 007.

[12] D. J. Gross and V. Rosenhaus, A generalization of SachdevYe-Kitaev, J. High Energy Phys. 02 (2017) 093.

[13] A. Kitaev and S. J. Suh, The soft mode in the Sachdev-YeKitaev model and its gravity dual, J. High Energy Phys. 05 (2018) 183.

[14] V. Rosenhaus, An introduction to the SYK model, arXiv:1807.03334.

[15] S. Giombi, I. R. Klebanov, and G. Tarnopolsky, Bosonic tensor models at large $N$ and small $\epsilon$, Phys. Rev. D 96, 106014 (2017).

[16] J. Murugan, D. Stanford, and E. Witten, More on supersymmetric and $2 \mathrm{~d}$ analogs of the SYK model, J. High Energy Phys. 08 (2017) 146.

[17] A. Dymarsky, I. R. Klebanov, and R. Roiban, Perturbative search for fixed lines in large $\mathrm{N}$ gauge theories, J. High Energy Phys. 08 (2005) 011.

[18] E. Pomoni and L. Rastelli, Large N field theory and AdS Tachyons, J. High Energy Phys. 04 (2009) 020.

[19] D. Grabner, N. Gromov, V. Kazakov, and G. Korchemsky, Strongly $\gamma$-Deformed $\mathcal{N}=4$ Supersymmetric Yang-Mills Theory as an Integrable Conformal Field Theory, Phys. Rev. Lett. 120, 111601 (2018).

[20] S. Prakash and R. Sinha, A complex fermionic tensor model in $d$ dimensions, J. High Energy Phys. 02 (2018) 086.

[21] P. Breitenlohner and D.Z. Freedman, Stability in gauged extended supergravity, Ann. Phys. (N.Y.) 144, 249 (1982).

[22] I. R. Klebanov and E. Witten, AdS/CFT correspondence and symmetry breaking, Nucl. Phys. B556, 89 (1999).

[23] J. Liu, E. Perlmutter, V. Rosenhaus, and D. SimmonsDuffin, $d$-dimensional SYK, AdS loops, and $6 j$ symbols, arXiv:1808.00612.
[24] K. Bulycheva, $\mathcal{N}=2$ SYK model in the superspace formalism, J. High Energy Phys. 04 (2018) 036.

[25] C.-M. Chang, S. Colin-Ellerin, and M. Rangamani, On melonic supertensor models, arXiv:1806.09903.

[26] H. Osborn and A. C. Petkou, Implications of conformal invariance in field theories for general dimensions, Ann. Phys. (N.Y.) 231, 311 (1994).

[27] S. Giombi, S. Prakash, and X. Yin, A note on CFT correlators in three dimensions, J. High Energy Phys. 07 (2013) 105.

[28] D. B. Kaplan, J.-W. Lee, D. T. Son, and M. A. Stephanov, Conformality lost, Phys. Rev. D 80, 125005 (2009).

[29] S. Giombi, I. R. Klebanov, and G. Tarnopolsky, Conformal $\mathrm{QED}_{d}, F$-Theorem and the $\epsilon$ expansion, J. Phys. A 49, 135403 (2016).

[30] V. Gorbenko, S. Rychkov, and B. Zan, Walking, weak firstorder transitions, and complex CFTs, arXiv:1807.11512.

[31] K. Bulycheva, I. R. Klebanov, A. Milekhin, and G. Tarnopolsky, Spectra of operators in large $N$ tensor models, Phys. Rev. D 97, 026016 (2018).

[32] G. Parisi, How to measure the dimension of the parton field, Nucl. Phys. B59, 641 (1973).

[33] C. G. Callan, Jr. and D. J. Gross, Bjorken scaling in quantum field theory, Phys. Rev. D 8, 4383 (1973).

[34] A. L. Fitzpatrick, J. Kaplan, D. Poland, and D. SimmonsDuffin, The analytic bootstrap and AdS superhorizon locality, J. High Energy Phys. 12 (2013) 004.

[35] Z. Komargodski and A. Zhiboedov, Convexity and liberation at large spin, J. High Energy Phys. 11 (2013) 140.

[36] J. A. Gracey, I. Jack, and C. Poole, The a-function in six dimensions, J. High Energy Phys. 01 (2016) 174.

[37] H. Osborn and A. Stergiou, Seeking fixed points in multiple coupling scalar theories in the $\varepsilon$ expansion, J. High Energy Phys. 05 (2018) 051.

[38] R. D. Pisarski, Fixed Point Structure of $\phi^{6}$ in ThreeDimensions at Large $N$, Phys. Rev. Lett. 48, 574 (1982).

[39] J.S. Hager, Six-loop renormalization group functions of $\mathrm{O}(\mathrm{n})$-symmetric $\phi^{6}$-theory and epsilon-expansions of tricritical exponents up to $\varepsilon^{3}$, J. Phys. A 35, 2703 (2002).

[40] R. Gurau, The complete $1 / N$ expansion of a SYKlike tensor model, Nucl. Phys. B916, 386 (2017).

[41] L. Fei, S. Giombi, and I. R. Klebanov, Critical $O(N)$ models in 6- $\epsilon$ dimensions, Phys. Rev. D 90, 025018 (2014).

[42] L. Fei, S. Giombi, I. R. Klebanov, and G. Tarnopolsky, Three loop analysis of the critical $O(N)$ models in $6-\epsilon$ dimensions, Phys. Rev. D 91, 045011 (2015).

[43] T. Azeyanagi, F. Ferrari, and F. I. Schaposnik Massolo, Phase Diagram of Planar Matrix Quantum Mechanics, Tensor, and Sachdev-Ye-Kitaev Models, Phys. Rev. Lett. 120, 061602 (2018).

[44] T. Azeyanagi, F. Ferrari, P. Gregori, L. Leduc, and G. Valette, More on the new large $D$ limit of matrix models, Ann. Phys. (Amsterdam) 393, 308 (2018).

[45] F. Ferrari, The large D limit of planar diagrams, arXiv: 1701.01171.

[46] S. Choudhury, A. Dey, I. Halder, L. Janagal, S. Minwalla, and R. Poojary, Notes on melonic $O(N)^{q-1}$ tensor models, J. High Energy Phys. 06 (2018) 094. 
[47] A. Almheiri and J. Polchinski, Models of $\mathrm{AdS}_{2}$ backreaction and holography, J. High Energy Phys. 11 (2015) 014.

[48] J. Maldacena, D. Stanford, and Z. Yang, Conformal symmetry and its breaking in two dimensional nearly Anti-deSitter space, Prog. Theor. Exp. Phys. 2016, 12C104 (2016).

[49] J. Engelsoy, T. G. Mertens, and H. Verlinde, An investigation of $\mathrm{AdS}_{2}$ backreaction and holography, J. High Energy Phys. 07 (2016) 139.

[50] K. Jensen, Chaos in $\mathrm{AdS}_{2}$ Holography, Phys. Rev. Lett. 117, 111601 (2016).
[51] F. Ferrari, V. Rivasseau, and G. Valette, A new large N expansion for general matrix-tensor models, arXiv:1709 .07366 .

[52] S. S. Gubser, C. Jepsen, Z. Ji, and B. Trundy, Higher melonic theories, J. High Energy Phys. 09 (2018) 049.

[53] L. Lionni and J. Thrigen, Multi-critical behaviour of 4-dimensional tensor models up to order 6, arXiv:1707 .08931 .

[54] I. R. Klebanov, F. Popov, and G. Tarnopolsky (to be published). 